\title{
Overall Damage Identification of Flag-shaped Hysteresis Systems under Seismic Excitation
}

\author{
Cong Zhou ${ }^{* 1}$, J. Geoffrey Chase ${ }^{1 a}$ \\ Geoffrey W. Rodgers ${ }^{1}$, Chao Xu' ${ }^{2}$ and Hamish Tomlinson ${ }^{1}$ \\ ${ }^{1}$ Department of Mechanical Engineering, University of Canterbury, \\ Private Bag 4800, Christchurch 8140, New Zealand \\ ${ }^{2}$ School of Astronautics, Northwestern Polytechnical University, \\ X'an 710072, China \\ (Received , Revised , Accepted )
}

\begin{abstract}
This research investigates the structural health monitoring of nonlinear structures after a major seismic event. It considers the identification of flag-shaped or pinched hysteresis behavior in response to structures as a more general case of a normal hysteresis curve without pinching. The method is based on the overall least squares methods and the log likelihood ratio test. In particular, the structural response is divided into different loading and unloading sub-half cycles. The overall least squares analysis is first implemented to obtain the minimum residual mean square estimates of structural parameters for each sub-half cycle with the number of segments assumed. The log likelihood ratio test is used to assess the likelihood of these nonlinear segments being true representations in the presence of noise and model error. The resulting regression coefficients for identified segmented regression models are finally used to obtain stiffness, yielding deformation and energy dissipation parameters. The performance of the method is illustrated using a single degree of freedom system and a suite of 20 earthquake records. RMS noise of 5\%, 10\%, $15 \%$ and $20 \%$ is added to the response data to assess the robustness of the identification routine. The proposed method is computationally efficient and accurate in identifying the damage parameters within $10 \%$ average of the known values even with $20 \%$ added noise. The method requires no user input and could thus be automated and performed in real-time for each sub-half cycle, with results available effectively immediately after an event as well as during an event, if required.
\end{abstract}

Keywords: structural health monitoring; flag-shaped hysteresis system; structural parameter identification; least squares; log likelihood ratio; seismic response; system identification; SHM

\section{Introduction}

Under conventional seismic design strategy, civil engineering structures are designed to undergo inelastic deformation to dissipate earthquake energy, which can lead to residual displacements. Residual deformation increases the repair cost and downtime, as well as the difficulty in recovering the structure to the initial position. To solve this deficiency, a large number of self-centering systems and devices, which exhibit a flag-shaped hysteretic behavior, have been

*Corresponding author, Ph.D. , E-mail: cong.zhou@pg.canterbury.ac.nz

a Professor, E-mail: geoff.chase@ canterbury.ac.nz 
developed to avoid residual deformation and provide energy dissipation capacity. The selfcentering system refers to the use of post-tensioned with stiffness, usually done with tendons associated with energy dissipation elements, to aid returning the system to its original position without external load and offer yielding to eliminate permanent deformation, such as posttensioned beam-to-column connections for moment-resisting steel frame (Christopoulos et al. 2002, Garlock et al. 2005, Ricles et al. 2001, Rodgers et al. 2008), steel brace dissipating elements (Bartera and Giacchetti 2004, Christopoulos et al. 2008, Tremblay et al. 2008) and shape memory alloy (SMA) seismic isolation devices (Alam et al. 2009, Attanasi et al. 2009, Casciati and Hamdaoui 2008, Ozbulut and Hurlebaus 2011). The seismic application of these flag-shaped hysteretic structures has increased since the 1994 Northridge earthquake in the United States and 1995 Hyogoken-Nambu earthquake in Japan. However, these structures can still experience various degrees or types of damage under extreme excitation. Real-time or rapid structural health monitoring (SHM) is suitable for determining the damage state of the structure, enabling a more optimum assessment and recovery planning after an earthquake event

Many current vibration-based SHM methods are based on the idea that modal parameters change, such as natural frequency, mode shapes and damping, as a result of structural damage (Doebling et al. 1996). However, these methods are not robust in the presence of noise and not accurate to localize damage (Chang et al. 2003). Furthermore, these methods are only applicable to structures where vibration response is linear (Chase et al. 2005a), which is not always the case for real structures after extreme earthquake.

Damage identification methods based on Eigensystem Realization Algorithm (ERA) methods are also commonly used (Bernal and Gunes 2000, Giraldo et al. 2004, Lus et al. 2003). The ERA method is based on knowledge of the time domain free response data. Flexibility-based methods were used to identify the changes in flexibility matrices to localize the damage in structure ((Bernal 2002, Bernal and Gunes 2004, Koo et al. 2008, Yan et al. 2009). The damage locating vectors in the null space of the flexibility change are estimated from output signals, without reference to a model of the structure, and then can be used to localize damage by inspecting zero stress fields over damaged regions. These off-line approaches require the entire measured response to process and identify structural damage. The results might not be immediately available after an event, especially if human input is required. Adaptive Ho filter techniques (Sato and Qi 1998) and Kalman filter methods (Lee and Yun 2008, Loh et al. 2000, Yang et al. 2006) can achieve real-time or rapid results. However, they have significant computational cost and complexity, and are better for linear systems.

Finally, real-time LMS-based methods have been used for a benchmark problem (Chase et al. 2005a), and also for a nonlinear rocking structure (Chase et al. 2005b). These methods can only track down changes in structural stiffness. A modified LMS-based method has also been used to identify changes in stiffness and plastic deformation (Nayyerloo et al. 2011). However, these LMS based methods are not accurate for nonlinear yielding structures, especially with complex hysteretic behavior.

This study develops a simplified method to identify the physical parameters that are directly related to structural health monitoring for a flag-shaped hysteretic SDOF system. The performance of the proposed method is demonstrated and validated using a simulated flag-shaped hysteretic system. The effect of measurement noise is investigated by adding 5\%, 10\%, 15\% and 20\% RMS noise to the measured response. The robustness of the method is evaluated using a suite of 20 different earthquake records. 


\section{The LMS and log likelihood test ratio method (LMSLL)}

\subsection{Equation of motion}

The equation of motion of a single degree of freedom (SDOF) system is defined as:

$$
m \ddot{x}+c \dot{x}+F(x)=-m \ddot{x}_{g}
$$

where $x, \dot{x}$ and $\ddot{x}$ are the displacement, velocity and acceleration of the SDOF system; $m$ is the mass; $F(x)$ is the restoring force of the hysteresis system; and $c$ is the viscous damping coefficient:

$$
c=\frac{4 m \pi \xi}{T}
$$

$\xi$ is the initial fraction of critical damping; $T$ is the time period of the system. Using Eq. (2), it can be obtained:

$$
F(x)=-m\left(\ddot{x}_{g}+\ddot{x}\right)-\frac{4 m \pi \xi}{T} \dot{x}
$$

In this equation, the acceleration $\ddot{x}$ and $\ddot{x}_{g}$, which is the ground acceleration, can be measured; the velocity and displacement are derived by integration and correction, or by applying a set of sensors and methods (Fu and Moosa 2002, Hann et al. 2009, Hwang et al. 2012, Psimoulis and Stiros 2008, Safak and Hudnut 2006, Smyth and Wu 2007, Zhou et al. 2013, Casciati and Fuggini 2011). Assuming $m, \xi$ and $T$ to be available from the basic knowledge of the system, the restoring force $F(x)$ is hence consequently obtained. Thus, the hysteresis loop of the system can be constructed.

\subsection{Hysteresis model}

Fig. 1(a) shows the flag-shaped force-displacement relationship that is representative of a selfcentering system. The parameters for this hysteretic model are $k_{e}, \alpha, \beta$ and $d_{y}$. The coefficient $k_{e}$ is the pre-yielding stiffness, $\alpha$ is the ratio of post-yielding stiffness to pre-yielding stiffness. The energy dissipation coefficient $\beta$ reflects the dissipation capacity. And $d_{y}$ is the yield displacement of the hysteresis system.

In general, if the hysteresis loop can be divided into each single segment by the turning points $x_{1}-x_{8}$, the linear regression analysis can then be applied to each segment for the identification of the physical parameters. The Rain Flow Counting method is a widely used way to divide the time history of structural response into a number of half cycles for the cumulative damage assessment (Powell and Allahabadi 1988). ). However, the half-cycles separated by Rain Flow method are not in chronological order but grouped with different deformation magnitude, which is not appropriate to track down the evolution of damage parameters overtime if degradation occurs. Therefore, the hysteresis loop are divided into many sub-half cycles that are in chronological order. In particular, the whole hysteretic response can be separated into many sub-half cycles according to the loading-unloading path turning point, such as $x_{4}$ and $x_{8}$, where the velocity is zero and the displacement is a local maximum or minimum (Xu et al. 2014).

All the sub-half cycles are then divided into four types of piecewise linear model with one, two, 
three or four segments, as shown in Fig. 1(b). If the numbers of segments of these piecewise linear models could be identified from the data, then the overall least squares solution (Hudson 1966) can be implemented. And therefore $k_{e}, \alpha, \beta$ and $d_{y}$ can be accurately defined.

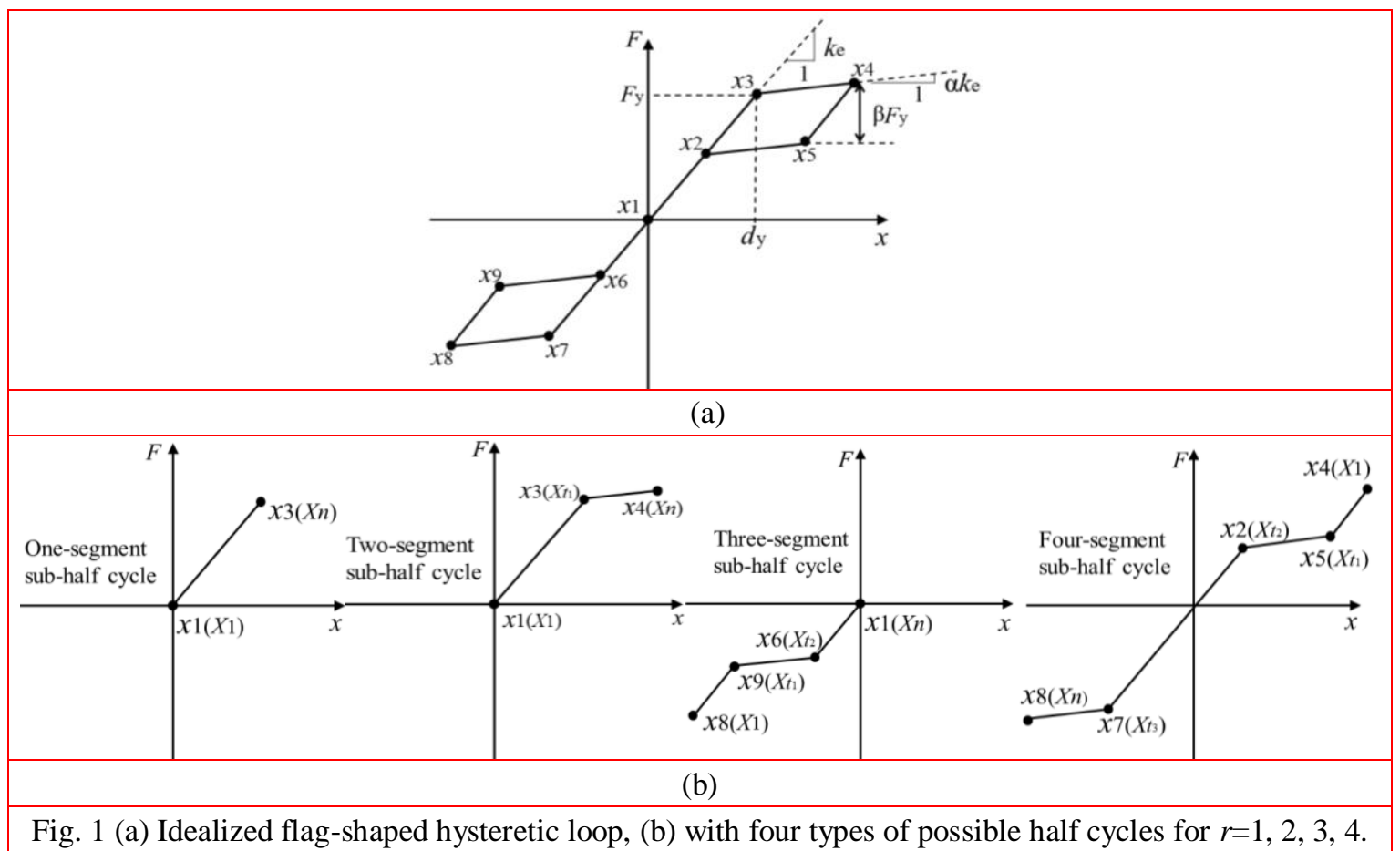

\subsection{Parameter identification procedures}

The assumed number of segments for the sub-half cycles is $r$, where $r=1,2,3$ or 4 , the $r$-phase linear model, as defined by (Hudson 1966), can be written as:

$$
\begin{aligned}
G(x) & =g_{1}(x)=a_{1} x+b_{1} & & X_{1} \leq x \leq X_{t_{1}} \\
& =g_{2}(x)=a_{2} x+b_{2} & & X_{t_{1}} \leq x \leq X_{t_{2}} \\
& =\cdots & & \\
& =g_{r}(x)=a_{r} x+b_{r} & & X_{t_{r-1}} \leq x \leq X_{n}
\end{aligned}
$$

where $X_{t_{1}}, \ldots, X_{t_{r-1}}$ are the breakpoints in the sub-half cycles, as shown in Fig. 1(b); $\left(X_{1}, Y_{1}\right), \ldots$, $\left(X_{n}, Y_{n}\right)$ are $n$ pairs of displacement and restoring force data during the sub-half cycles, and can be represented by:

$$
Y_{i}=G\left(X_{i}\right)+e_{i} \quad i=1, \ldots, n
$$

where $e_{i}$ are the random errors caused by measurement noise or model uncertainty. Suppose $e_{i}$ are normally and independently distributed with zero mean and standard deviation $\sigma^{2}$. Then the 
overall residual sum of squares for an $r$-phase linear model is determined as:

$$
R_{r}=\sum_{i=1}^{t_{1}}\left[Y_{i}-g_{1}\left(X_{i}\right)\right]^{2}+\sum_{i=t_{1}+1}^{t_{2}}\left[Y_{i}-g_{2}\left(X_{i}\right)\right]^{2}+\cdots+\sum_{i=t_{r-1}+1}^{n}\left[Y_{i}-g_{r}\left(X_{i}\right)\right]^{2}
$$

The optimum approximate solution to the $r$-phase linear model is to determine the best estimate values of $X_{t_{1}}, \ldots, X_{t_{r-1}}$ in order to minimize $R_{r}$.

In this model, the derivative equal to zero of Eq. (6) cannot be used here due to the discontinuous nature of the turning points. Thus, the data is divided into every feasible $r$ groups. For each $r$ groups, standard linear regression is implemented in every segment, in order to obtain the model coefficients $a_{1}, b_{1}, \ldots, a_{r}, b_{r}(r=1,2,3,4)$. Assuming that the overall model is continuous, at each joint point between segments in the loop, the breakpoints $\left(X_{t_{1}}, \ldots, X_{t_{r-1}}\right)$ are computed by:

$$
X_{t_{i}}=\frac{b_{i+1}-b_{i}}{a_{i}-a_{i+1}}
$$

And the residual sum of squares is obtained whenever $X_{i} \leq X_{t_{i}} \leq X_{i+1}$. Then the overall solution is the value of $X_{t_{1}}, \ldots, X_{t_{r-1}}$ that correspond to the smallest value of $R_{r}$ in Eq. (6).

The likelihood-ratio chi square test is used to test each of the sub-half cycles. The likelihood ratio $\lambda$ is defined as:

$$
\lambda=\left(\frac{R_{r+1}}{R_{r}}\right)^{\frac{n}{2}} \quad r=1,2,3,4
$$

The hypothesis test is performed between $\left[H_{0}\right.$ : there are $r$ segments in this piecewise linear model] and $\left[H_{1}\right.$ : there are $r+1$ segments in the model]. Then the large sample distribution of the likelihood ratio defined as $-2 \log \lambda$ is a chi-squared distribution with $2(r+1)$ degrees of freedom when the null hypothesis is true (Feder 1975, Quandt 1958). Therefore, the rejection of $H_{0}$ in favor of $H_{1}$ is true whenever:

$$
-2 \log \lambda \geq \chi_{\varepsilon}^{2}(k)
$$

where $\varepsilon$ is the significance level, and is set to a low value in order to reduce the probability of committing an error by rejecting $H_{0}$ when it is true (Walpole et al. 2011), and $k$ is the number of degrees of freedom of the chi-squared distribution. The critical value of $\chi_{\varepsilon}^{2}(k)$ can be found in statistical tables (Walpole et al. 2011).

In this study, the significance level $\varepsilon$ is set to 0.001 and $k=2(r+1)$ for an $r$ phase model identification. If the value of the likelihood ratio is less than $\chi_{\varepsilon}^{2}(k)$, hence there is no evidence against $H_{0}$, and it is concluded that the sub-half cycle is an $r$ phase model.

Using this two-step method, the number of segments for all sub-half cycles are identified, and the breakpoints $\left(X_{t_{1}}, \ldots, X_{t_{r-1}}\right)$ and regression coefficients $\left(a_{1}, b_{1}, \ldots, a_{r}, b_{r}\right)$ for each sub-half cycle are obtained. And therefore the physical parameters of the flag-shaped hysteretic system are defined. In particular:

For the pre-yielding stiffness $k_{e}$ and post-yielding stiffness $k_{p}$ : 


$$
\begin{aligned}
k_{e} & =a_{1} \quad \text { for } r=1,2 \\
& =a_{1} \text { and } a_{3} \text { for } r=3,4 \\
k_{p} & =a_{2} \quad \text { for } r=2,3 \\
& =a_{2} \text { and } a_{4} \text { for } r=4
\end{aligned}
$$

For the yield displacement $d_{y}$ :

$$
d_{y}=\left|X_{t_{3}}\right| \quad \text { for } r=4
$$

And the energy dissipation coefficient $\beta$ is defined as:

$$
\beta=\left|\frac{Y_{1}-Y_{t_{1}}}{Y_{n}}\right|=\left|\frac{\left(a_{1} X_{1}+b_{1}\right)-\left(a_{2} X_{t_{1}}+b_{2}\right)}{a_{4} X_{t_{3}}+b_{4}}\right| \quad \text { for } r=4
$$

The yielding absorbed hysteretic energy is computed for each three-segment $(r=3)$ and foursegment ( $r=4)$ sub-half cycles (Chopra 2001):

$$
E_{Y}=\sqrt{\left(Y_{1}-Y_{t_{1}}\right)^{2}+\left(X_{1}-X_{t_{1}}\right)^{2}} \sqrt{\left(Y_{t_{1}}-Y_{t_{2}}\right)^{2}+\left(X_{t_{1}}-X_{t_{2}}\right)^{2}}\left|\frac{a_{1}-a_{2}}{\sqrt{\left(a_{1}^{2}+1\right)+\left(a_{2}^{2}+1\right)}}\right|
$$

\section{Summary of the proposed method:}

Step 1) Assume $r=1$ and $r=2$ for all the sub-half cycles respectively, and compute $R_{1}, a_{1}$ and $b_{1}$ for $r=1$, and $R_{2}, X_{t_{1}}, a_{1}, b_{1}, a_{2}$ and $b_{2}$ for $r=2$ using Eqs. (4)-(7).

Step 2) Compute the likelihood ratio $-2 \log \lambda$ for every sub-half cycle using Eq. (8), and identify the linear $(r=1)$ sub-half cycles by Eq. (9), i.e. $-2 \log \lambda<\chi_{0.001}^{2}(4)=18.47$ (Walpole et al. 2011).

Step 3) Assume $r=3$ for all the nonlinear sub-half cycles, and compute $R_{3}, X_{t_{1}}, X_{t_{2}}, a_{1}, b_{1}, a_{2}$, $b_{2}, a_{3}$ and $b_{3}$ for $r=3$. Then compute $-2 \log \lambda$ and get the two segment $(r=2)$ sub-half cycles by $-2 \log \lambda<\chi_{0.001}^{2}(6)=22.46$.

Step 4) Assume $r=4$ for the unidentified sub-half cycles, and compute $R_{4}, X_{t_{1}}, X_{t_{2}}, X_{t_{3}}, a_{1}, b_{1}$, $a_{2}, b_{2}, a_{3}, b_{3}, a_{4}$ and $b_{4}$ for $r=4$. Compute $-2 \log \lambda$ and identify the three segment ( $r=3$ ) sub-half cycles using $-2 \log \lambda<\chi_{0.001}^{2}(8)=26.12$. The remaining sub-half cycles must then be four segment $(r=4)$ sub-half cycles.

Step 5) Estimate the physical parameters $k_{e}, k_{p}, d_{y}$ and $\beta$ by Eqs. (10)-(14) for all the sub-half cycles identified in steps 1-4. 


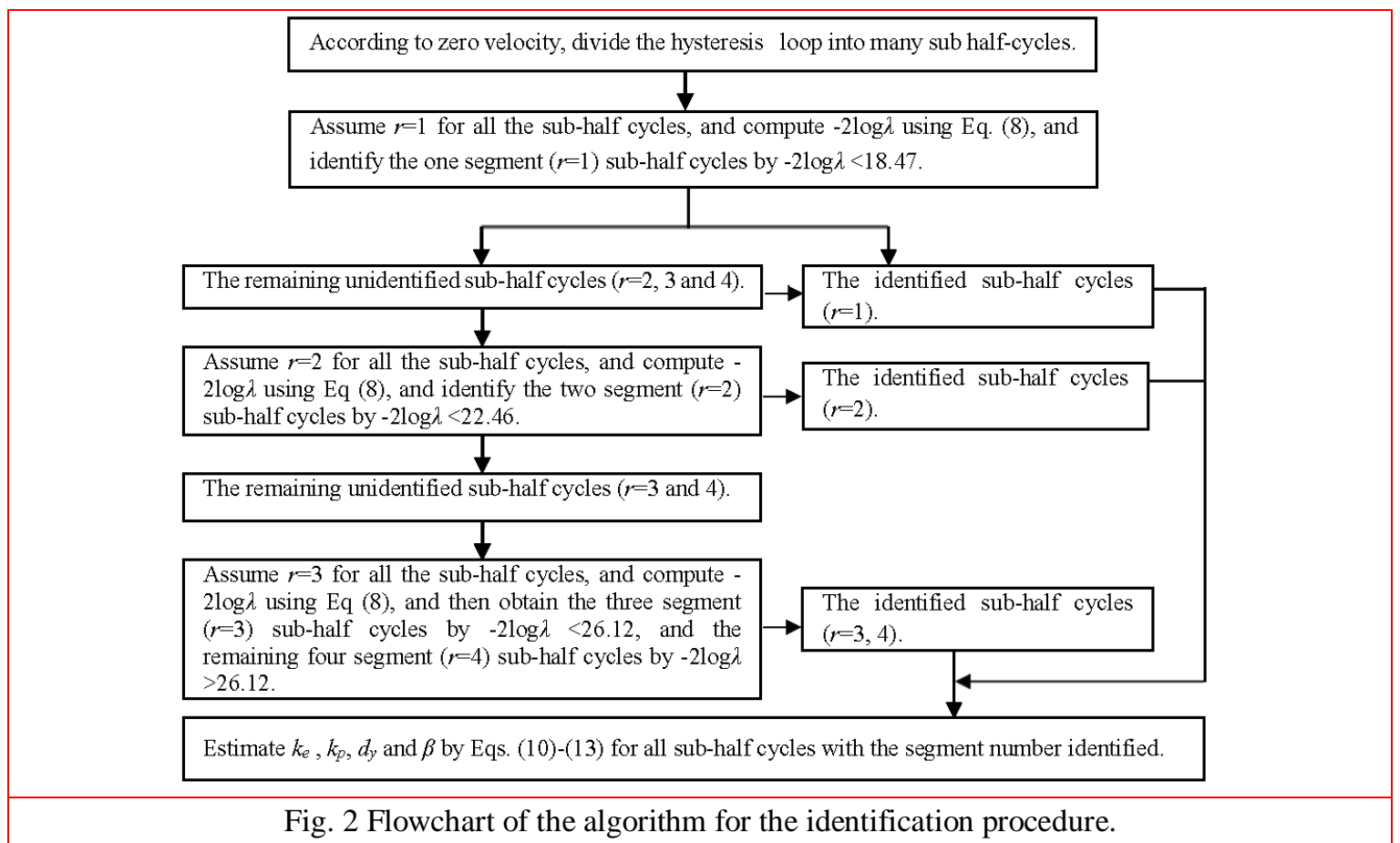

Fig. 2 shows the flowchart of the algorithm for the identification procedure. Once the hysteresis loop can be constructed using the measurement data, which has already been reality (Iwan 2002, Iwan et al. 2013), the whole procedure to identify the segment number $r$ for each sub-half cycle can be processed without user input. Finally, the physical parameters $k_{e}, k_{p}, d_{y}$ and $\beta$ can be estimated by Eqs. (10)-(13).

\section{Simulated proof-of-concept structure}

The simulated proof-of-concept structure is a SDOF system that is representative of a seven storey steel moment resisting frames (MRFs) incorporating post-tensioned energy dissipating (PTED) connections at all beam-to-column connections and at the base of each column. The PTED connections incorporates high strength steel post-tensioned bars designed to remain elastic during the seismic response, and confined energy-dissipation bars designed to yield both in tension and compression. Thus, this steel MRFs structural behaviour can be achieved without introducing residual drift during the seismic response, and the flag-shaped hysteretic model is considered to represent the hysteretic behaviour of this system (Christopoulos et al. 2002).

This fixed base steel MRFs system founded on soil type D is designed according to the seismic provisions of the 1997 edition of the uniform building code (UBC 1997). Each story has $3.4 \mathrm{~m}$ height, and the seismic weight of the system is $4000 \mathrm{kN}$ that result in the first time period of $1.0 \mathrm{~s}$ and the pre-yielding stiffness ke of $157.9 \mathrm{kN} / \mathrm{mm}$. The post-yielding stiffness $\mathrm{kp}$ is $23.68 \mathrm{kN} / \mathrm{mm}$ with the post-yielding stiffness coefficient $\alpha$ set to be 0.15 . The energy coefficient $\beta$ is set to be 0.5 and the yield displacement dy is $24.85 \mathrm{~mm}$ for the steel MRFs with PTED connections founded on soil type D. In addition, a 5\% damping, which is commonly adopted by design codes and 
standards (Atkinson and Pierre 2004, Pekcan et al. 1999), is considered in simulating the structural response.

The proposed identification procedure was implemented in Matlab. The simulated structure was subjected to the 1989 Loma Prieta earthquake Hollister Differential Array record with peak ground acceleration (PGA) of $0.269 \mathrm{~g}$. The system acceleration response was simulated using the Newmark- $\beta$ integration method. Different levels of random RMS noise was added to the simulated acceleration measurements to provide a more realistic measurement situation. The displacement and velocity were estimated using a low-frequency-measured displacement corrected acceleration integration method (Hann et al. 2009). In this case study, the low-frequency-measured displacement was taken at $1 \mathrm{~Hz}$ and acceleration data was taken at $1000 \mathrm{~Hz}$.

To assess the robustness of the proposed method over different ground motions, a suite of 20 different earthquake events that are representative of ordinary earthquakes having a probability of exceedance of $10 \%$ in 50 years are used to generate the hysteretic loop of the simulated structure. These records were recorded on soil types C or D, the PGA range from $0.116 \mathrm{~g}$ to $0.417 \mathrm{~g}$, and the hypocentre distance range between 13 and $25 \mathrm{~km}$. More details of these 20 earthquakes records can be found in the reference (Christopoulos et al. 2002). These earthquake records can be downloaded from the PEER strong motion database (PEER 2005). The same model parameters were used for all of the records and 5\%,10\%, 15\% and 20\% RMS noise were added to the simulated acceleration and displacement response measurements.

Finally, the segment numbers $(r=1,2,3,4)$ of all the sub-half cycles were identified and the physical parameters of the structure were obtained in each case using the overall least squares solution.

During the earthquake excitation, some sub-half cycles exhibited narrow, almost negligible plastic or nonlinear behavior. These small plastic responses that is difficult to find the correct slope, can significantly affect the accuracy of the estimated post-yielding stiffness $\left(k_{p}\right)$. Thus, the slope of plastic segment for those nonlinear sub-half cycles is used to ignore these narrow sub-half cycles for the estimation of the post-yielding stiffness only when the plastic deformation $\Delta d p$ calculated from Eq. (15) is more than an optimal threshold value $\Delta d$.

$$
\begin{aligned}
\Delta d p & =\quad\left|X_{t_{2}}-X_{t_{1}}\right| \quad \text { for } r=2,3 \\
& =\left|X_{t_{2}}-X_{t_{1}}\right| \text { and }\left|X_{t_{4}}-X_{t_{3}}\right| \quad \text { for } r=4
\end{aligned}
$$

However, some small amplitude plastic cycles could be missing when this threshold is used. The hysteretic dissipation energy by a large number of small amplitude can significantly exceed that dissipated up to failure through the application of a few large amplitude cycles (Teran-Gilmore et al. 2003, Teran-Gilmore and Jirsa 2005). Therefore, the effect of ignoring these sub-half cycles on the results was investigated by varying the threshold of $\Delta d$.

Performance is assessed by accuracy in recovering the true model values in the presence of noise over several events. The impact of thresholds used to ensure significant nonlinear motion $(r=2,3,4)$ in identified half cycles is also assessed. The overall analyses assess both performance accuracy and robustness to noise providing a range of trade offs.

\section{Results and discussion}




\subsection{Identificaiton of the number of segments of sub-half cycles}

Fig. 3 shows the identification results for one segment $(r=1)$ sub-half cycles for the Loma Prieta event. The values of $-2 \log \lambda$ for the sub-half cycles (\#11-14, 17, 19-28), with variable noise levels are less than $\chi_{0.001}^{2}(4)=18.47$ and rejection is not permitted according to model hypothesis Eq. (9). Thus, the sub-half cycles below the rejection value, as shown in Fig. 3(a), are identified as one segment $(r=1)$ linear models or simply linear structural responses. And the sub-half cycles in Fig. 3 (b) that are not fitted well by one $(r=1)$ segment model are processed to the next identification step, as shown in Fig. 4.

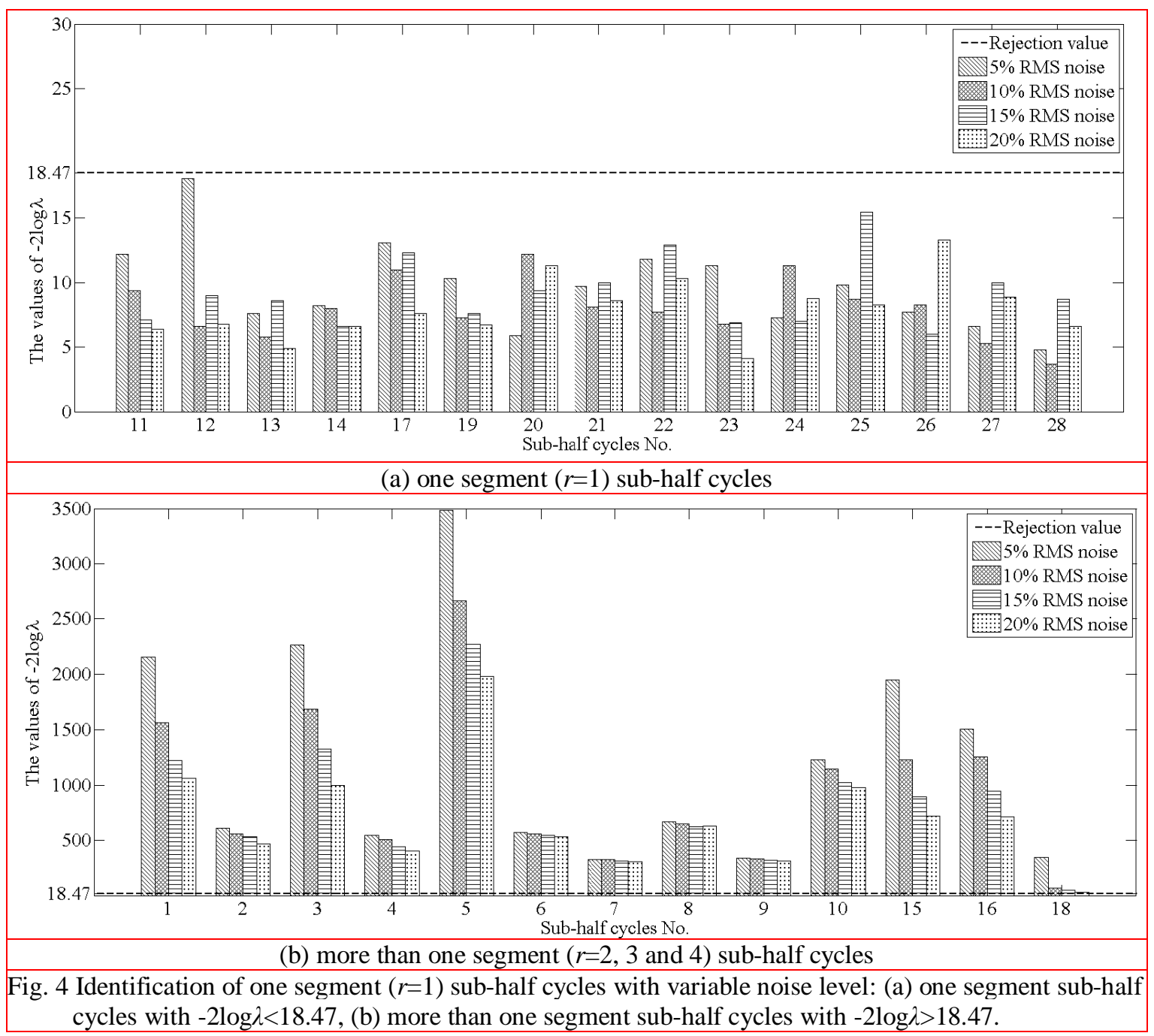




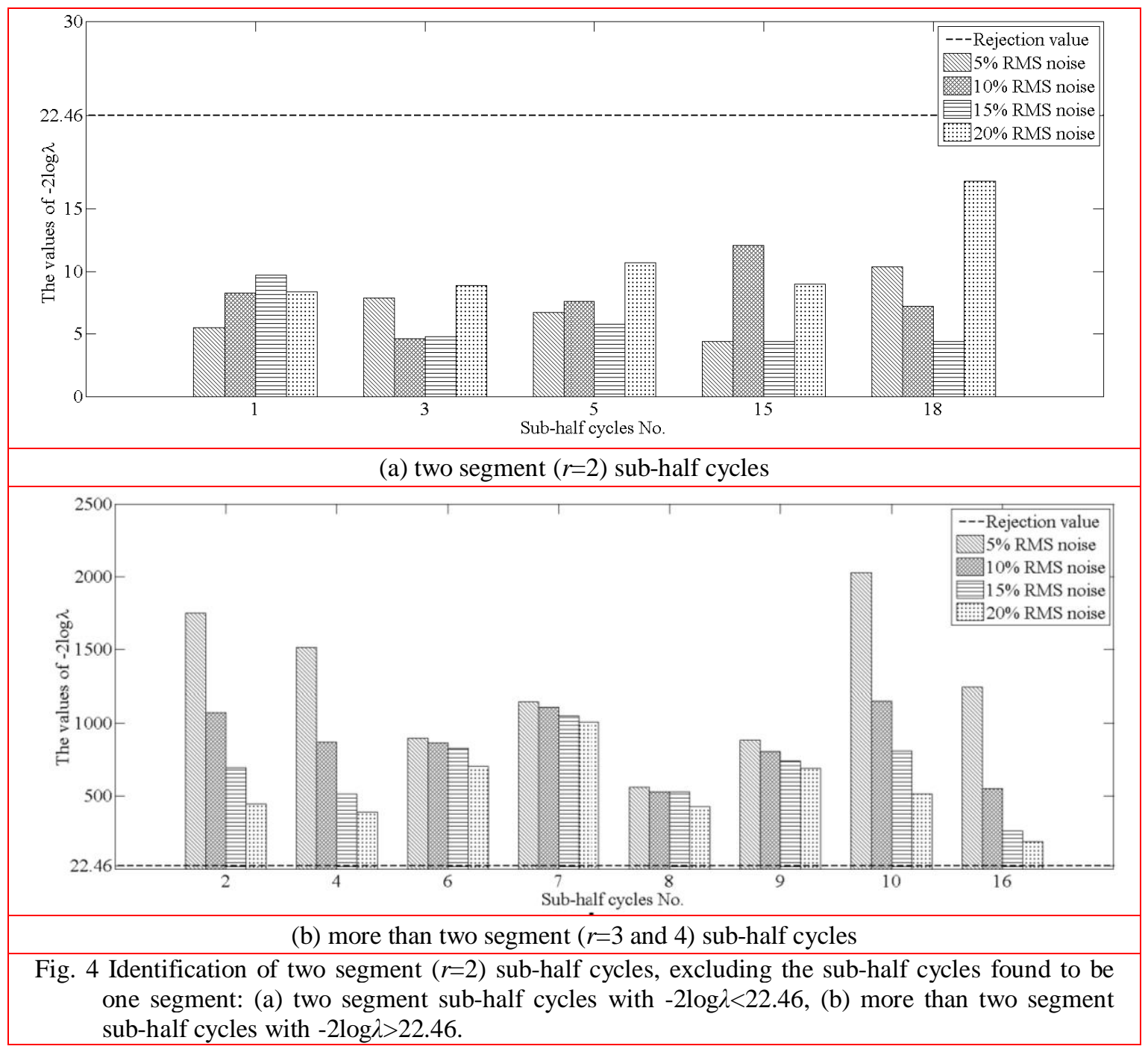

Fig. 4 shows the identification results for two segment $(r=2)$ sub-half cycles, excluding the subhalf cycles found to be one segment $(r=1)$ in Fig. 3(a). The sub-half cycles in Fig. 4(a) are below the rejection value of $\chi_{0.001}^{2}(6)=22.46$, and thus are identified as two segment $(r=2)$. The identification results for the unidentified sub-half cycles in Fig. 4(b) are shown in Fig. 5. It can be seen from Fig. 5(a) that the three-segment $(r=3)$ sub-half cycles are identified using the values of $2 \log \lambda$ below the rejection value of $\chi_{0.001}^{2}(8)=26.12$. And the remained sub-half cycle in Fig. 5(b) are then identified as four segment with a full flag shaped response. 


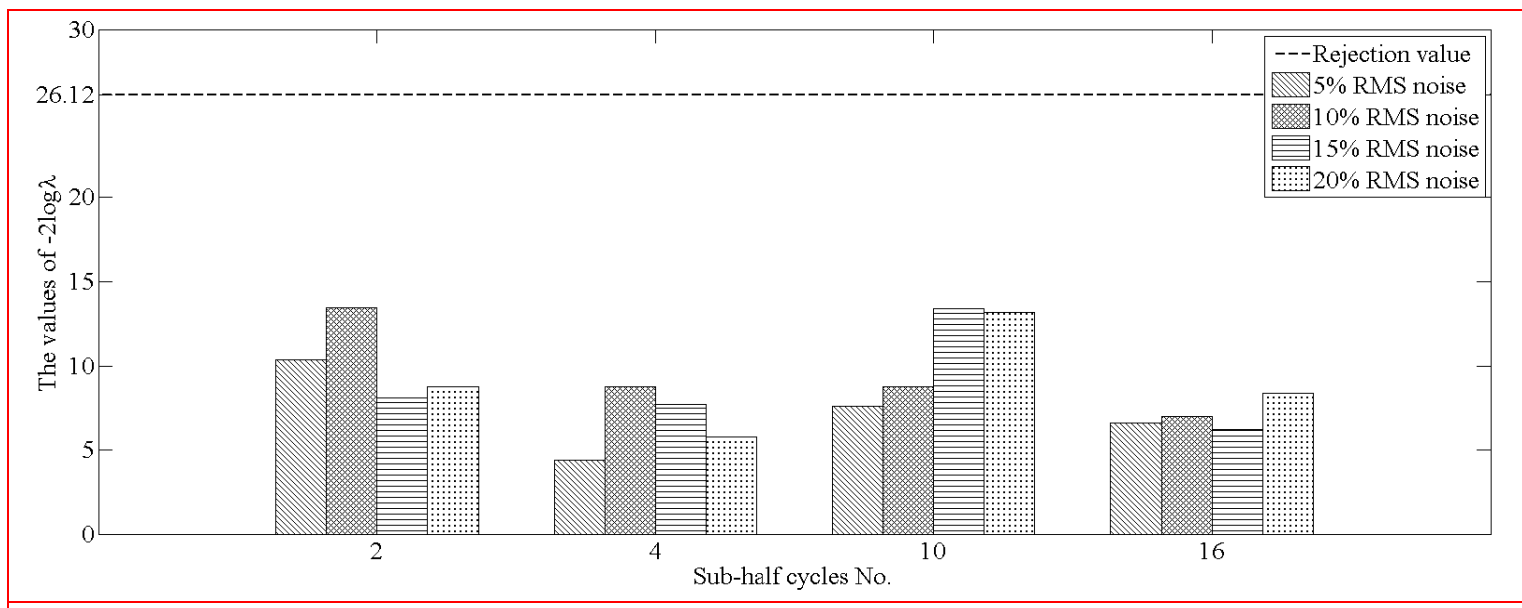

(a) three segment $(r=3)$ sub-half cycles

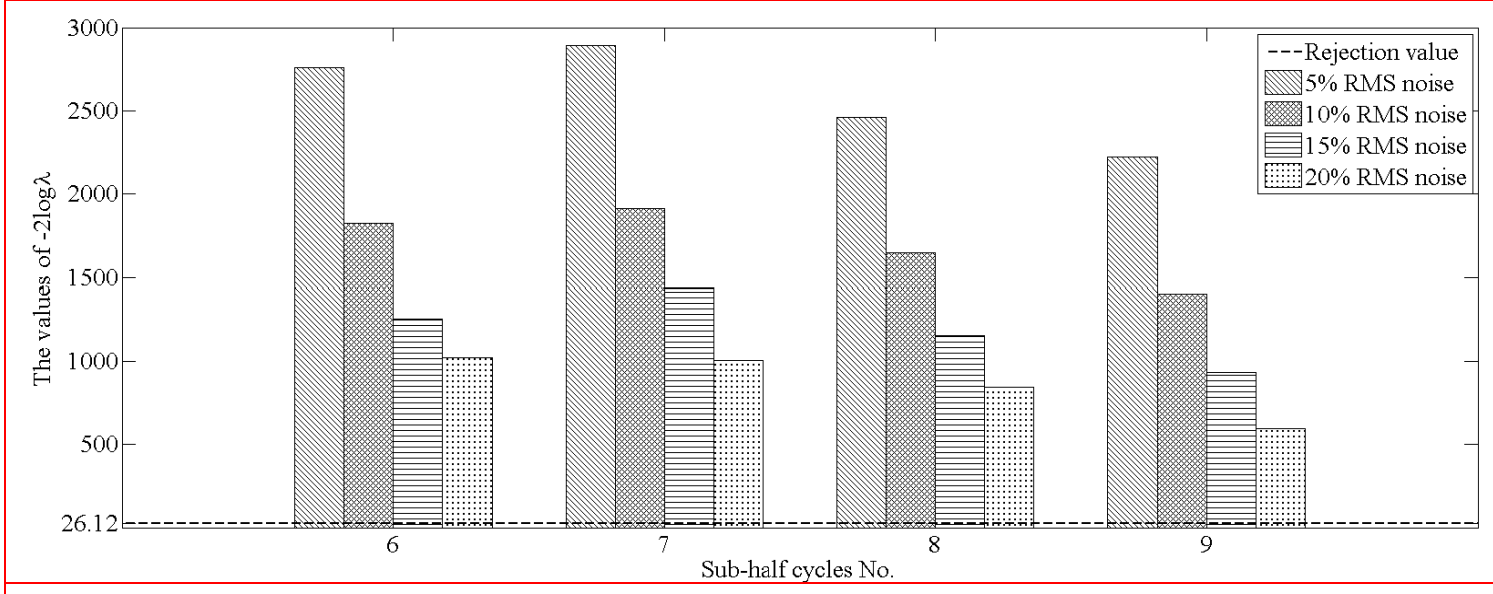

(b) four segment $(r=4)$ sub-half cycles

Fig. 5 Identification of three segment $(r=3)$ sub-half cycles, excluding one and two segment sub-half cycles: (a) three segment sub-half cycles with $-2 \log \lambda<26.12$, (b) four segment half cycles with $-2 \log \lambda>26.12$.

It also can be seen from Figs. 3(a)-5(a) that the values of -2log $\lambda$ for the sub-half cycles that are fitted well by $r$ segment model, vary essentially randomly with increasing noise. However, the values of $-2 \log \lambda$ for the sub-half cycles that are not well fitted by that value of $r$, as shown in Figs. $3(b)-5(b)$, decrease when the added noise is increasing. The $-2 \log \lambda$ represents the difference degree between the assumed $r$ and $r+1$ segment models. This difference degree are very significant, with the values of $-2 \log \lambda$ very large and above the rejection value, when the sub-half cycles cannot fitted well by the assumed $r$ segment model. However, with the increasing noise, the true state of the sub-half cycle is more discrete and the difference between $r$ and $r+1$ segment models is less distinguishable. Thus, the values of $-2 \log \lambda$ representing the difference degree are also decreasing when the added noise is increased.

However, the difference between the assumed $r$ and $r+1$ segment models is very small when the sub-half cycles can be fitted well by the $r$ segment model. Even with the increasing noise, this difference is also very small. Therefore, the variation of the values of $-2 \log \lambda$ for these sub-half cycles is mainly caused by the randomness of the added noise. 


\subsection{Effect of threshold}

Fig. 6 shows the estimates of pre-yielding stiffness and post-yielding stiffness with different thresholds of $\Delta d$ for $5 \%, 10 \%, 15 \%$ and $20 \%$ added noise. For variable thresholds at the same noise level, the pre-yielding stiffness $k_{e}$ is robust because the elastic parts for all the sub-half cycles are big enough to obtain good estimates. The post-yielding stiffness $k_{p}$ is not robust because of the influence of relatively very small cycles when the threshold is low. It also can be seen that the estimates of stiffness $k_{e}$ and $k_{p}$ vary with noise, and good estimates can be obtained using high thresholds to ensure significant nonlinear motion even at $20 \%$ noise level.

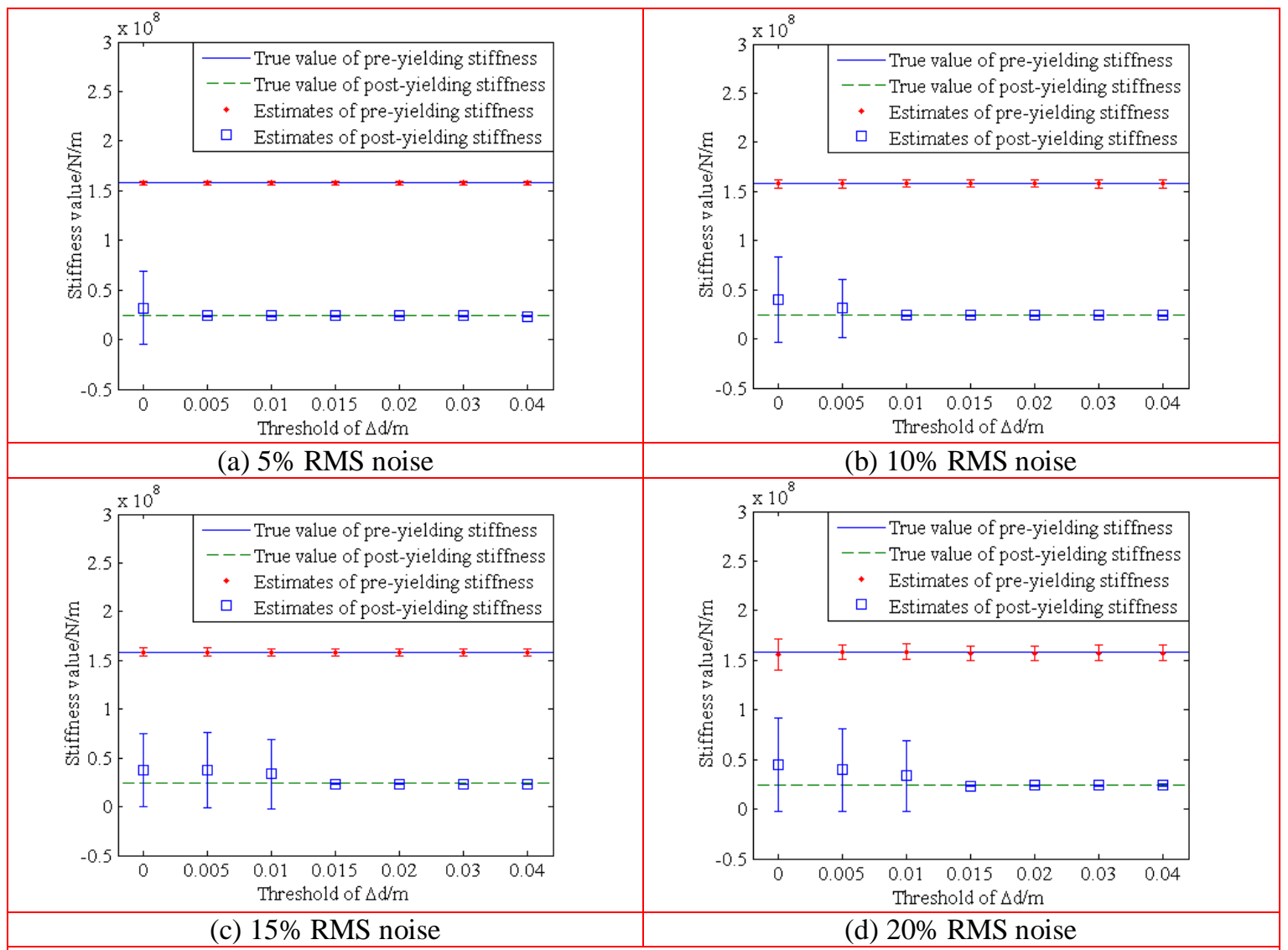

Fig. 6 Estimates results of stiffness, (a) at 5\% RMS noise, (b) at 10\% RMS noise, (c) at 15\% RMS noise, (d) at $20 \%$ RMS noise. The error bars show the mean and one standard deviation over all sub-half cycles.

Figs. 7 shows the estimates of yield displacement $d_{y}$ and Fig. 8 shows the energy dissipation coefficient $\beta$. The results are robust with varying thresholds because the turning points used to compute the estimates of $d_{y}$ and $\beta$ are not affected by small cycles. The results also give a good approximation of the true input with errors within 5\% even at $20 \%$ added noise. In addition, the 
standard deviation shows a smaller increase with increasing noise indicating a low sensitivity to noise.
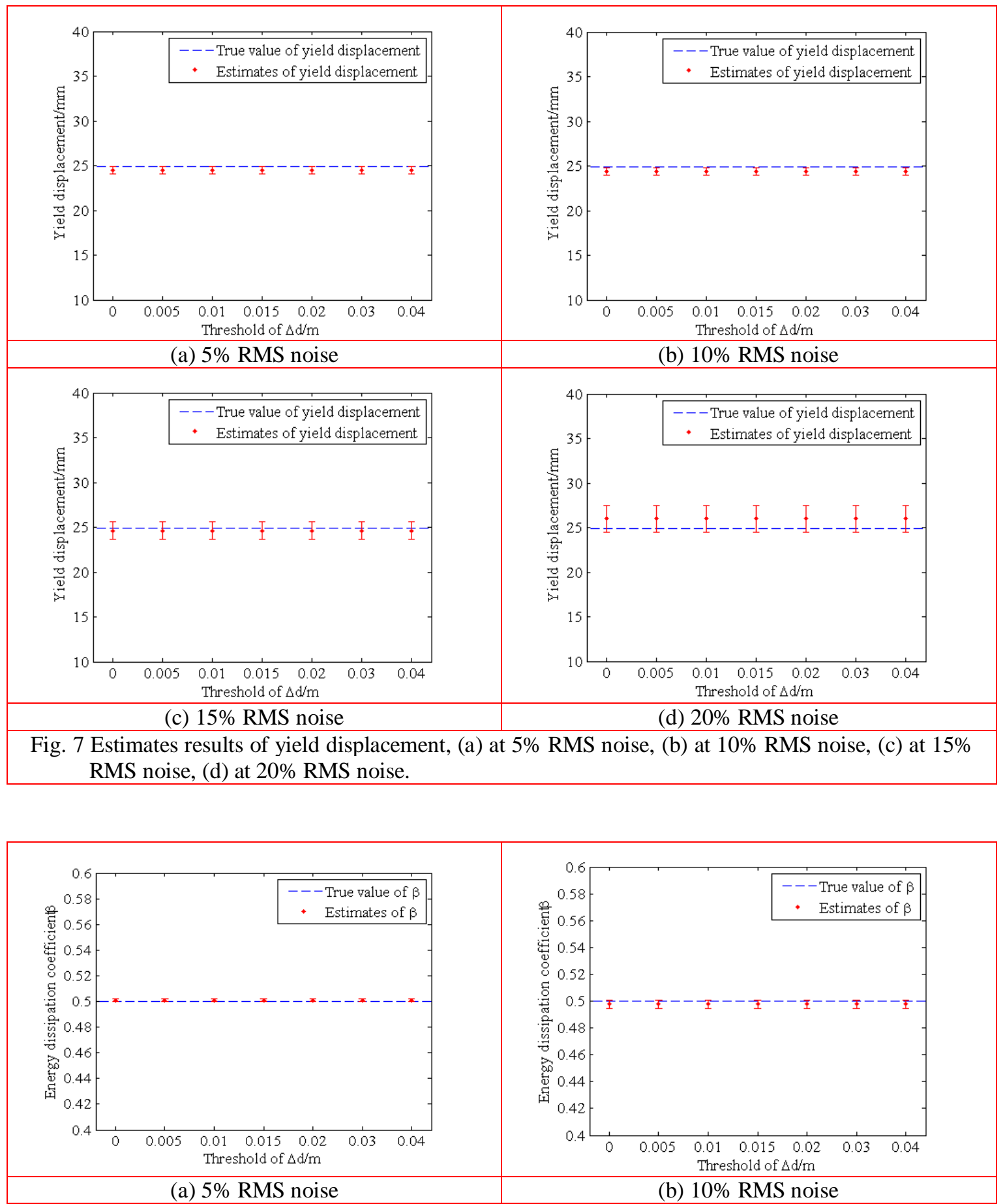

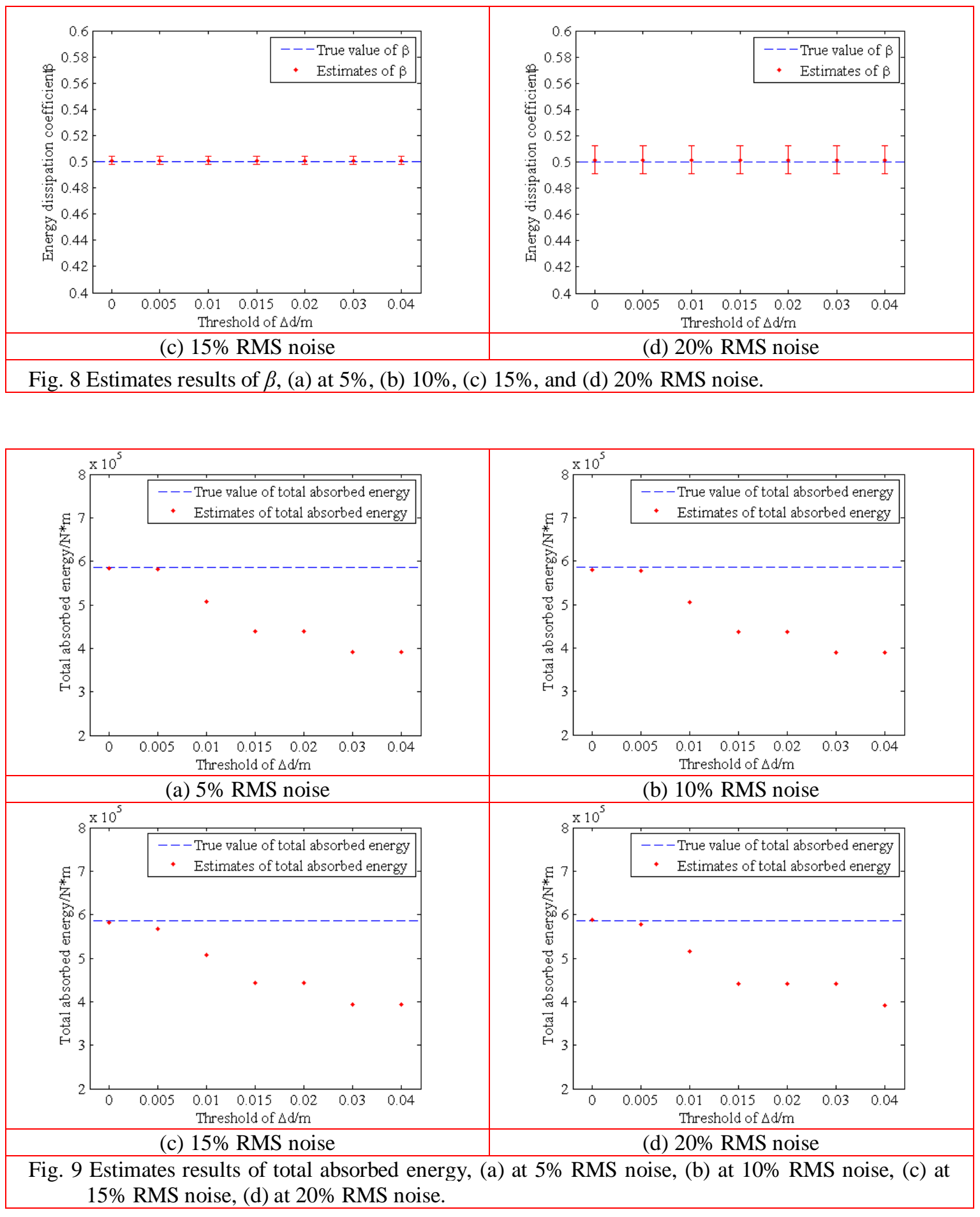

Fig. 9 shows the estimates of total absorbed hysteretic energy. It can be seen that the total energy dissipation shows a low sensitivity to different noise level at the same threshold because all 
four panels are almost the same. In addition, the total energy dissipation dropped as more cycles are ignored with the rising threshold at the same noise level. The result is expected and thus no threshold $(\Delta d=0)$ should be used in calculating this value so that damage or low cycle fatigue assessment is not affected. Importantly, using large thresholds to estimate $k_{e}, k_{p}, d_{y}$ and $\beta$, with zero thresholds for dissipated energy, is computationally simple and efficient once all sub-half cycles are identified.

\subsection{Results for 20 different earthquake records}

As a result, a threshold $\Delta d=0.04$ is chosen to evaluate the results of a 20 suite earthquake records with 5\%, 10\%, 15\% and 20\% added RMS noise, as shown in Tables 1-4, respectively. A '-' represent elastic behavior during the entire earthquake.

It can be seen from Tables 1-4 that the structure was identified as remaining linear during EQ1, $3,6,9,10,12,14,17$ and 19 when the structural response of the system is only linear during the whole earthquake event, which indicate the method is robustness to different ground motion. The mean estimates of $k_{e}, k_{p}, d_{y}$ and $\beta$ across all sub-half cycles of an event at different levels of noise matched well with the true input parameters $\left(k_{e}=157.9, k_{p}=23.7, d_{y}=24.9, \beta=0.5\right)$. Although the errors of the mean estimates of $k_{e}, k_{p}, d_{y}$ and $\beta$ show a small increase with increasing added noise, the average errors of the estimates of $k_{e}, k_{p}, d_{y}$ and $\beta$ are within $3.6 \%$ even at $20 \%$ noise level.

It also can be seen that the coefficient of variation (COV) of $k_{e}, k_{p}, d_{y}$ and $\beta$ are very small at $5 \%$ added noise, and the COV increases with increasing noise, as might be expected. However, the maximum COV is $18.7 \%$ of the mean value even with $20 \%$ added noise, with most within $10 \%$, which is practically speaking a good result given uncertainty in construction and degradation over time.

Table 1 Results for 20 different earthquake events with $5 \%$ noise

\begin{tabular}{|c|c|c|c|c|c|c|c|c|}
\hline \multirow{2}{*}{$\begin{array}{c}\text { Earthquake } \\
\text { Record }\end{array}$} & \multicolumn{2}{|c|}{$k_{e}=157.9(\mathrm{kN} / \mathrm{mm})$} & \multicolumn{2}{c|}{$k_{p}=23.7(\mathrm{kN} / \mathrm{mm})$} & \multicolumn{2}{c|}{$d_{y}=24.9(\mathrm{~mm})$} & \multicolumn{2}{c|}{$\beta=0.500$} \\
\cline { 2 - 10 } & Mean & $\mathrm{COV}$ & Mean & COV & Mean & COV & Mean & COV \\
\hline EQ1 & 157.8 & $0.5 \%$ & - & - & - & - & - & - \\
\hline EQ2 & 157.8 & $1.2 \%$ & 23.8 & $2.3 \%$ & 25.0 & $3.8 \%$ & 0.486 & $8.4 \%$ \\
\hline EQ3 & 157.9 & $0.4 \%$ & - & - & - & - & - & - \\
\hline EQ4 & 158.2 & $1.8 \%$ & 23.8 & $1.0 \%$ & 24.4 & $3.9 \%$ & 0.500 & $2.5 \%$ \\
\hline EQ5 & 158.3 & $0.6 \%$ & 24.0 & $1.1 \%$ & 24.7 & $3.3 \%$ & 0.501 & $2.1 \%$ \\
\hline EQ6 & 157.6 & $0.8 \%$ & - & - & - & - & - & - \\
\hline EQ7 & 157.7 & $0.6 \%$ & 23.7 & $1.9 \%$ & 24.7 & $2.2 \%$ & 0.494 & $4.1 \%$ \\
\hline EQ8 & 157.3 & $1.0 \%$ & 23.9 & $1.8 \%$ & 24.9 & $2.6 \%$ & 0.501 & $1.5 \%$ \\
\hline EQ9 & 157.8 & $0.3 \%$ & - & - & - & - & - & - \\
\hline EQ10 & 157.8 & $0.5 \%$ & - & - & - & - & - & - \\
\hline EQ11 & 157.6 & $1.1 \%$ & 23.8 & $2.3 \%$ & 24.7 & $3.4 \%$ & 0.485 & $11.5 \%$ \\
\hline EQ12 & 158.0 & $0.6 \%$ & - & - & - & - & - & - \\
\hline EQ13 & 157.5 & $1.2 \%$ & 23.7 & $0.6 \%$ & 24.9 & $2.0 \%$ & 0.499 & $0.8 \%$ \\
\hline EQ14 & 157.9 & $0.3 \%$ & - & - & - & - & - & - \\
\hline EQ15 & 157.9 & $0.8 \%$ & 23.8 & $0.3 \%$ & 24.5 & $1.3 \%$ & 0.499 & $0.1 \%$ \\
\hline EQ16 & 158.1 & $1.2 \%$ & 23.8 & $0.8 \%$ & 24.9 & $3.5 \%$ & 0.499 & $1.7 \%$ \\
\hline EQ17 & 157.9 & $0.4 \%$ & - & - & - & - & - & - \\
\hline EQ18 & 157.9 & $1.4 \%$ & 23.6 & $0.9 \%$ & 24.6 & $4.1 \%$ & 0.498 & $1.9 \%$ \\
\hline EQ19 & 158.0 & $0.5 \%$ & - & - & - & - & - & - \\
\hline EQ20 & 158.4 & $1.1 \%$ & 23.8 & $0.7 \%$ & 24.7 & $3.6 \%$ & 0.499 & $1.3 \%$ \\
\hline
\end{tabular}


Table 2 Results for 20 different earthquake events with $10 \%$ noise

\begin{tabular}{|c|c|c|c|c|c|c|c|c|}
\hline \multirow{2}{*}{$\begin{array}{c}\text { Earthquake } \\
\text { Record }\end{array}$} & \multicolumn{2}{|c|}{$k_{e}=157.9(\mathrm{kN} / \mathrm{mm})$} & \multicolumn{2}{c|}{$k_{p}=23.7(\mathrm{kN} / \mathrm{mm})$} & \multicolumn{2}{c|}{$d_{y}=24.9(\mathrm{~mm})$} & \multicolumn{2}{c|}{$\beta=0.500$} \\
\cline { 2 - 10 } & Mean & $\mathrm{COV}$ & Mean & $\mathrm{COV}$ & Mean & COV & Mean & COV \\
\hline EQ1 & 157.5 & $0.8 \%$ & - & - & - & - & - & - \\
\hline EQ2 & 157.5 & $1.6 \%$ & 24.4 & $7.7 \%$ & 24.7 & $3.7 \%$ & 0.500 & $1.4 \%$ \\
\hline EQ3 & 157.9 & $0.6 \%$ & - & - & - & - & - & - \\
\hline EQ4 & 157.7 & $4.1 \%$ & 23.9 & $1.8 \%$ & 24.6 & $11.8 \%$ & 0.498 & $5.4 \%$ \\
\hline EQ5 & 157.0 & $1.9 \%$ & 22.9 & $6.7 \%$ & 22.9 & $13.1 \%$ & 0.504 & $2.9 \%$ \\
\hline EQ6 & 157.2 & $1.8 \%$ & - & - & - & - & - & - \\
\hline EQ7 & 158.7 & $1.9 \%$ & 24.3 & $3.5 \%$ & 24.6 & $3.0 \%$ & 0.496 & $1.5 \%$ \\
\hline EQ8 & 157.2 & $0.9 \%$ & 24.2 & $6.4 \%$ & 25.1 & $7.2 \%$ & 0.503 & $2.3 \%$ \\
\hline EQ9 & 158.2 & $0.7 \%$ & - & - & - & - & - & - \\
\hline EQ10 & 157.9 & $0.9 \%$ & - & - & - & - & - & - \\
\hline EQ11 & 158.5 & $4.1 \%$ & 23.8 & $5.0 \%$ & 24.7 & $4.5 \%$ & 0.476 & $11.8 \%$ \\
\hline EQ12 & 157.5 & $1.1 \%$ & - & - & - & - & - & - \\
\hline EQ13 & 157.7 & $1.7 \%$ & 23.6 & $1.4 \%$ & 24.7 & $4.5 \%$ & 0.495 & $3.2 \%$ \\
\hline EQ14 & 158.0 & $0.6 \%$ & - & - & - & - & - & - \\
\hline EQ15 & 158.8 & $2.4 \%$ & 23.3 & $1.7 \%$ & 25.0 & $3.4 \%$ & 0.496 & $1.5 \%$ \\
\hline EQ16 & 157.3 & $1.5 \%$ & 23.7 & $2.3 \%$ & 24.9 & $6.8 \%$ & 0.492 & $4.0 \%$ \\
\hline EQ17 & 157.9 & $0.8 \%$ & - & - & - & - & - & - \\
\hline EQ18 & 157.2 & $1.6 \%$ & 23.7 & $2.6 \%$ & 24.8 & $4.4 \%$ & 0.502 & $2.1 \%$ \\
\hline EQ19 & 157.6 & $1.1 \%$ & - & - & - & - & - & - \\
\hline EQ20 & 157.9 & $2.6 \%$ & 23.5 & $3.3 \%$ & 24.9 & $4.0 \%$ & 0.479 & $19.5 \%$ \\
\hline
\end{tabular}

Table 3 Results for 20 different earthquake events with $15 \%$ noise

\begin{tabular}{|c|c|c|c|c|c|c|c|c|}
\hline \multirow{2}{*}{$\begin{array}{c}\text { Earthquake } \\
\text { Record }\end{array}$} & \multicolumn{2}{|c|}{$k_{e}=157.9(\mathrm{kN} / \mathrm{mm})$} & \multicolumn{2}{c|}{$k_{p}=23.7(\mathrm{kN} / \mathrm{mm})$} & \multicolumn{2}{c|}{$d_{y}=24.9(\mathrm{~mm})$} & \multicolumn{2}{c|}{$\beta=0.500$} \\
\cline { 2 - 10 } & Mean & COV & Mean & COV & Mean & COV & Mean & COV \\
\hline EQ1 & 157.7 & $1.0 \%$ & - & - & - & - & - & - \\
\hline EQ2 & 158.1 & $3.3 \%$ & 23.6 & $4.5 \%$ & 24.5 & $7.3 \%$ & 0.488 & $4.3 \%$ \\
\hline EQ3 & 158.0 & $1.7 \%$ & - & - & - & - & - & - \\
\hline EQ4 & 156.4 & $5.5 \%$ & 24.4 & $4.6 \%$ & 24.8 & $10.1 \%$ & 0.483 & $8.1 \%$ \\
\hline EQ5 & 157.6 & $2.8 \%$ & 22.5 & $6.3 \%$ & 24.4 & $4.5 \%$ & 0.484 & $4.8 \%$ \\
\hline EQ6 & 158.2 & $2.1 \%$ & - & - & - & - & - & - \\
\hline EQ7 & 158.3 & $2.5 \%$ & 23.3 & $1.7 \%$ & 25.0 & $5.2 \%$ & 0.502 & $2.4 \%$ \\
\hline EQ8 & 154.1 & $2.1 \%$ & 23.4 & $3.1 \%$ & 24.7 & $6.9 \%$ & 0.502 & $5.4 \%$ \\
\hline EQ9 & 157.7 & $0.8 \%$ & - & - & - & - & - & - \\
\hline EQ10 & 157.5 & $1.8 \%$ & - & - & - & - & - & - \\
\hline EQ11 & 156.9 & $5.8 \%$ & 23.5 & $4.3 \%$ & 25.7 & $10.1 \%$ & 0.481 & $13.6 \%$ \\
\hline EQ12 & 157.1 & $2.4 \%$ & - & - & - & - & - & - \\
\hline EQ13 & 158.3 & $3.1 \%$ & 23.9 & $2.0 \%$ & 25.2 & $4.4 \%$ & 0.489 & $3.1 \%$ \\
\hline EQ14 & 157.4 & $1.2 \%$ & - & - & - & - & - & - \\
\hline EQ15 & 157.2 & $2.8 \%$ & 24.1 & $4.9 \%$ & 24.8 & $5.6 \%$ & 0.500 & $1.3 \%$ \\
\hline EQ16 & 158.0 & $2.7 \%$ & 24.0 & $6.3 \%$ & 24.2 & $10.3 \%$ & 0.508 & $4.5 \%$ \\
\hline EQ17 & 157.9 & $1.4 \%$ & - & - & - & - & - & - \\
\hline EQ18 & 157.1 & $3.1 \%$ & 23.9 & $3.2 \%$ & 25.1 & $7.2 \%$ & 0.500 & $2.8 \%$ \\
\hline EQ19 & 157.8 & $1.5 \%$ & - & - & - & - & - & - \\
\hline EQ20 & 158.1 & $3.5 \%$ & 23.4 & $3.2 \%$ & 25.4 & $8.3 \%$ & 0.485 & $5.6 \%$ \\
\hline
\end{tabular}


Table 4 Results for 20 different earthquake events with $20 \%$ noise

\begin{tabular}{|c|c|c|c|c|c|c|c|c|}
\hline \multirow{2}{*}{$\begin{array}{c}\text { Earthquake } \\
\text { Record }\end{array}$} & \multicolumn{2}{|c|}{$k_{e}=157.9(\mathrm{kN} / \mathrm{mm})$} & $k_{p}=23.7$ & $(\mathrm{kN} / \mathrm{mm})$ & \multicolumn{2}{c|}{$d_{y}=24.9(\mathrm{~mm})$} & \multicolumn{2}{c|}{$\beta=0.500$} \\
\cline { 2 - 10 } & Mean & COV & Mean & COV & Mean & COV & Mean & COV \\
\hline EQ1 & 157.8 & $1.3 \%$ & - & - & - & - & - & - \\
\hline EQ2 & 156.6 & $4.0 \%$ & 24.5 & $14.8 \%$ & 25.8 & $6.6 \%$ & 0.506 & $2.5 \%$ \\
\hline EQ3 & 158.0 & $1.0 \%$ & - & - & - & - & - & - \\
\hline EQ4 & 157.3 & $7.5 \%$ & 23.7 & $2.2 \%$ & 26.1 & $9.6 \%$ & 0.511 & $7.7 \%$ \\
\hline EQ5 & 156.8 & $3.0 \%$ & 26.1 & $11.3 \%$ & 24.7 & $6.5 \%$ & 0.506 & $6.2 \%$ \\
\hline EQ6 & 158.2 & $2.7 \%$ & - & - & - & - & - & - \\
\hline EQ7 & 157.8 & $2.5 \%$ & 25.1 & $7.5 \%$ & 23.9 & $5.9 \%$ & 0.499 & $3.1 \%$ \\
\hline EQ8 & 154.4 & $3.4 \%$ & 23.7 & $9.0 \%$ & 24.8 & $12.9 \%$ & 0.491 & $10.4 \%$ \\
\hline EQ9 & 157.3 & $1.3 \%$ & - & - & - & - & - & - \\
\hline EQ10 & 157.6 & $2.2 \%$ & - & - & - & - & - & - \\
\hline EQ11 & 154.9 & $5.3 \%$ & 23.9 & $3.2 \%$ & 23.8 & $11.3 \%$ & 0.472 & $18.7 \%$ \\
\hline EQ12 & 157.2 & $2.7 \%$ & - & - & - & - & - & - \\
\hline EQ13 & 158.0 & $3.7 \%$ & 24.1 & $3.1 \%$ & 24.8 & $10.1 \%$ & 0.474 & $10.7 \%$ \\
\hline EQ14 & 158.2 & $1.0 \%$ & - & - & - & - & - & - \\
\hline EQ15 & 157.6 & $3.0 \%$ & 23.5 & $4.3 \%$ & 23.3 & $10.7 \%$ & 0.498 & $2.8 \%$ \\
\hline EQ16 & 157.4 & $3.3 \%$ & 23.6 & $7.5 \%$ & 24.2 & $9.9 \%$ & 0.507 & $4.6 \%$ \\
\hline EQ17 & 157.8 & $1.6 \%$ & - & - & - & - & - & - \\
\hline EQ18 & 155.2 & $7.1 \%$ & 24.5 & $5.2 \%$ & 25.6 & $7.4 \%$ & 0.503 & $3.7 \%$ \\
\hline EQ19 & 157.5 & $3.0 \%$ & - & - & - & - & - & - \\
\hline EQ20 & 157.0 & $6.0 \%$ & 23.9 & $6.1 \%$ & 25.3 & $9.1 \%$ & 0.498 & $4.8 \%$ \\
\hline
\end{tabular}

Finally, the results show that the stiffness value $k_{e}$ and/or $k_{p}$ for each sub-half cycle in chronological order can be identified accurately. Thus, the evolution of stiffness value over time can be track down if degradation occurs. In addition, if the values of stiffness degradation exceed pre-defined design or safety limits, an alarm or other notice can be provided immediately to emergency response and/or the owners and managers of the structure without detailed engineering analysis.

\section{Conclusions}

This research develops a simple method for the damage identification of a highly nonlinear flag-shaped hysteretic structure. A simulated system with variable levels of added noise is used to demonstrate the feasibility of the method. The results show that a high threshold can yield a good estimates of post-yielding stiffness, and the estimation of pre-yielding stiffness, yielding displacement and energy dissipation coefficient are robust to different threshold. Good estimates of total hysteretic energy dissipation can be obtained when the threshold is not used. Given the computational simplicity of the method, the result can be evaluated with and without thresholds to ensure quality metrics are obtained from the final step of the method.

The sensitivity analysis shows good robustness of the method with the average coefficient of variation within $10 \%$ to different level noise and a range of 20 earthquake events. The accuracy of the method is also validated by identifying the structure as linear when the structural response of the system is only linear during the whole ground motion. In addition, the method can be extended to multi degree of freedom systems if the hysteresis loops are generated from measurement data 
obtained each floor. However, the robustness of the method to real data is still unproven since real data with significantly dynamic and plastic is very limited in availability. Thus, the proposed identification procedure remains to be experimentally validated and further tested before implementation in the field for final performance evaluation.

Finally, the method is computationally simple and can be implemented automatically without requiring human input. Thus, a rapid assessment can be made to offer significant information about structural damage and safety after an event.

\section{Acknowledgments}

The China Scholarship Council (No. 201306260119) and the University of Canterbury in support of this study is greatly acknowledged.

\section{References}

Alam, M.S., Youssef, M. and Nehdi, M. (2009), "Seismic performance of concrete frame structures reinforced with superelastic shape memory alloys", Smart Struct Syst, 5(5), 565-585.

Atkinson, G.M. and Pierre, J.-R. (2004), "Ground-motion response spectra in eastern North America for different critical damping values", Seismol. Res. Lett., 75(4), 541-545.

Attanasi, G., Auricchio, F. and Fenves, G.L. (2009), "Feasibility assessment of an innovative isolation bearing system with shape memory alloys", J. Earthquake Eng., 13(S1), 18-39.

Bartera, F. and Giacchetti, R. (2004), "Steel dissipating braces for upgrading existing building frames", $J$. Constr. Steel Res., 60(3), 751-769.

Bernal, D. (2002), "Load vectors for damage localization", J. Eng. Mech., 128(1), 7-14.

Bernal, D. and Gunes, B. (2004), "Flexibility based approach for damage characterization: benchmark application", J. Eng. Mech., 130(1), 61-70.

Bernal, D. and Gunes, B. (2000), "Observer/Kalman and subspace identification of the UBC benchmark structural model", Proceedings of the 14th ASCE Engineering Mechanics Conference, Texas, 21-24.

Casciati, F. and Fuggini, C. (2011), "Monitoring a steel building using GPS sensors", Smart Struct Syst, 7(5), $349-363$

Casciati, S. and Hamdaoui, K. (2008), "Experimental and numerical studies toward the implementation of shape memory alloy ties in masonry structures", Smart Struct Syst, 4(2), 153-169.

Chang, P.C., Flatau, A. and Liu, S. (2003), "Review paper: health monitoring of civil infrastructure", Struct. Health Monit., 2(3), 257-267.

Chase, J.G., Leo Hwang, K., Barroso, L. and Mander, J. (2005a), "A simple LMS-based approach to the structural health monitoring benchmark problem", Earthquake Engineering and Structural Dynamics, 34(6), 575-594.

Chase, J.G., Spieth, H.A., Blome, C.F. and Mander, J. (2005b), "LMS-based structural health monitoring of a non-linear rocking structure", Earthquake Engineering and Structural Dynamics, 34(8), 909-930.

Chopra, A.K. (2001), Dynamics of Structures: Theory and Applications to Earthquake Engineering, Prentice Hall, Englewood Cliffs, New Jersey, USA.

Christopoulos, C., Filiatrault, A. and Folz, B. (2002), "Seismic response of self-centring hysteretic SDOF systems", Earthquake Engineering and Structural Dynamics, 31(5), 1131-1150.

Christopoulos, C., Filiatrault, A., Uang, C.-M. and Folz, B. (2002), "Posttensioned energy dissipating connections for moment-resisting steel frames", J. Struct. Eng., 128(9), 1111-1120.

Christopoulos, C., Tremblay, R., Kim, H.-J. and Lacerte, M. (2008), "Self-centering energy dissipative bracing system for the seismic resistance of structures: development and validation", J. Struct. Eng., 134(1), 96-107.

Doebling, S.W., Farrar, C.R., Prime, M.B. and Shevitz, D.W. (1996), Damage Identification and Health 
Monitoring of Structural and Mechanical Systems from Changes in Their Vibration Characteristics: a Literature Review, Los Alamos National Lab, New Mexico, USA.

Feder, P.I. (1975), "The log likelihood ratio in segmented regression", The Annals of Statistics, 3(1), 84-97.

Fu, G. and Moosa, A.G. (2002), "An optical approach to structural displacement measurement and its application", J. Eng. Mech., 128(5), 511-520.

Garlock, M.M., Ricles, J.M. and Sause, R. (2005), "Experimental studies of full-scale posttensioned steel connections", J. Struct. Eng., 131(3), 438-448.

Giraldo, D., Yoshida, O., Dyke, S.J. and Giacosa, L. (2004), "Control-oriented system identification using ERA", Struct. Contr. Health Monit., 11(4), 311-326.

Hann, C.E., Singh-Levett, I., Deam, B.L., Mander, J.B. and Chase, J.G. (2009), "Real-time system identification of a nonlinear four-story steel frame structure - application to structural health monitoring", Sensors Journal, IEEE, 9(11), 1339-1346.

Hudson, D.J. (1966), "Fitting segmented curves whose join points have to be estimated", J. Amer. Statistical Assoc., 61(316), 1097-1129.

Hwang, J., Yun, H., Park, S.-K., Lee, D. and Hong, S. (2012), "Optimal methods of RTKGPS/Accelerometer integration to monitor the displacement of structures", Sensors, 12(1), 1014-1034.

International Conference of Building Officials. (1997), Uniform Building Code, Whittier,CA,USA.

Iwan, W.D. (2002), "R-SHAPE: a real-time structural health and performance evaluation system", Proceedings of the US Europe Workshop on Sensors and Smart Structures Technology, 33-38.

Iwan, W.D., Radulescu, D.C. and Radulescu, C. (2013), Extreme event performance evaluation using realtime hysteresis monitoring, US Patent 8,538,734.

Koo, K.-Y., Lee, J.-J., Yun, C.-B. and Kim, J.-T. (2008), "Damage detection in beam-like structures using deflections obtained by modal flexibility matrices", Smart Struct Syst, 4(5), 605-628.

Lee, K.J. and Yun, C.B. (2008), "Parameter identification for nonlinear behavior of RC bridge piers using sequential modified extended Kalman filter", Smart Struct Syst, 4(3), 319-342.

Loh, C.-H., Lin, C.-Y. and Huang, C.-C. (2000), "Time domain identification of frames under earthquake loadings", J. Eng. Mech., 126(7), 693-703.

Lus, H., Betti, R., Yu, J. and De Angelis, M. (2003), "Investigation of a system identification methodology in the context of the ASCE benchmark problem", J. Eng. Mech., 130(1), 71-84.

Nayyerloo, M., Chase, J., MacRae, G. and Chen, X. (2011), "LMS-based approach to structural health monitoring of nonlinear hysteretic structures", Struct. Health Monit., 10(4), 429-444.

Ozbulut, O.E. and Hurlebaus, S. (2011), "Seismic assessment of bridge structures isolated by a shape memory alloy/rubber-based isolation system", SmMaS, 20(1), 015003.

PEER. (2005), http://peer.berkeley.edu/smcat.

Pekcan, G., Mander, J.B. and Chen, S.S. (1999), "Fundamental considerations for the design of non-linear viscous dampers", Earthquake Eng. Struct. Dyn, 28(11), 1405-1425.

Powell, G.H. and Allahabadi, R. (1988), "Seismic damage prediction by deterministic methods: concepts and procedures", Earthquake Eng. Struct. Dyn., 16(5), 719-734.

Psimoulis, P.A. and Stiros, S.C. (2008), "Experimental assessment of the accuracy of GPS and RTS for the determination of the parameters of oscillation of major structures", CACAIE, 23(5), 389-403.

Quandt, R.E. (1958), "The estimation of the parameters of a linear regression system obeying two separate regimes", J. Amer. Statistical Assoc., 53(284), 873-880.

Ricles, J.M., Sause, R., Garlock, M.M. and Zhao, C. (2001), "Posttensioned seismic-resistant connections for steel frames", J. Struct. Eng., 127(2), 113-121.

Rodgers, G.W., Solberg, K.M., Chase, J.G., Mander, J.B., Bradley, B.A., Dhakal, R.P. and Li, L. (2008), "Performance of a damage-protected beam-column subassembly utilizing external HF2V energy dissipation devices", Earthquake Eng. Struct. Dyn., 37(13), 1549-1564.

Safak, E. and Hudnut, K. (2006), "Real-time structural monitoring and damage detection by acceleration and GPS sensors", 8th US National Conference on Earthquake Engineering, San Francisco, California, April.

Sato, T. and Qi, K. (1998), "Adaptive Ho filter: its application to structural identification", J. Eng. Mech., 
124(11), 1233-1240.

Smyth, A. and Wu, M. (2007), "Multi-rate Kalman filtering for the data fusion of displacement and acceleration response measurements in dynamic system monitoring", MSSP, 21(2), 706-723.

Teran-Gilmore, A., Avila, E. and Rangel, G. (2003), "On the use of plastic energy to establish strength requirements in ductile structures", Eng. Struct., 25(7), 965-980.

Teran-Gilmore, A. and Jirsa, J.O. (2005), "A damage model for practical seismic design that accounts for low cycle fatigue", Earthquake Spectra, 21(3), 803-832.

Tremblay, R., Lacerte, M. and Christopoulos, C. (2008), "Seismic response of multistory buildings with selfcentering energy dissipative steel braces", J. Struct. Eng., 134(1), 108-120.

Walpole, R.E., Myers, R.H. and Myers, S.L. (2011), Probability and Statistics for Engineers and Scientists, Pearson Prentice Hall, Upper Saddle River.

Xu, C., Chase, J.G. and Rodgers, G.W. (2014), "Physical parameter identification of nonlinear base-isolated buildings using seismic response data", Comput \& Struct, 145(1), 47-57.

Yan, G., Duan, Z. and Ou, J. (2009), "Damage detection for truss or frame structures using an axial strain flexibility", Smart Struct Syst, 5(3), 291-316.

Yang, J.N., Lin, S., Huang, H. and Zhou, L. (2006), "An adaptive extended Kalman filter for structural damage identification", Struct. Contr. Health Monit., 13(4), 849-867.

Zhou, C., Li, H.-N., Li, D.-S., Lin, Y.-X. and Yi, T.-H. (2013), "Online damage detection using pair cointegration method of time-varying displacement.", Smart Struct Syst, 12(3), 309-325. 


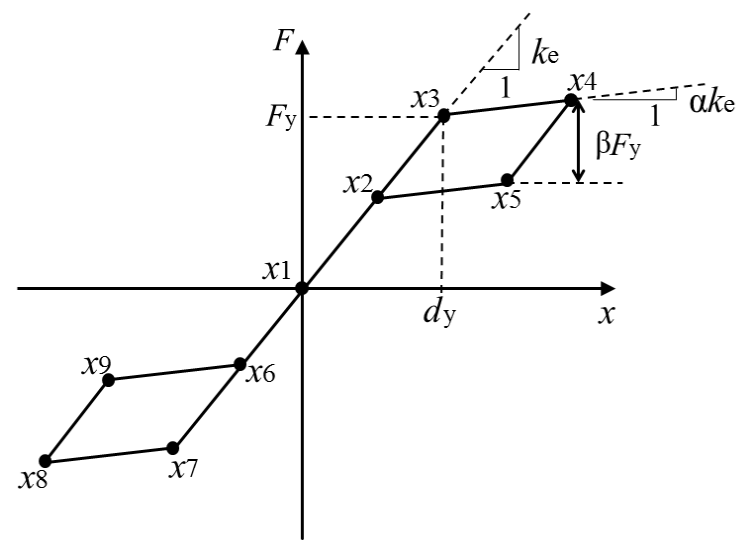

(a)
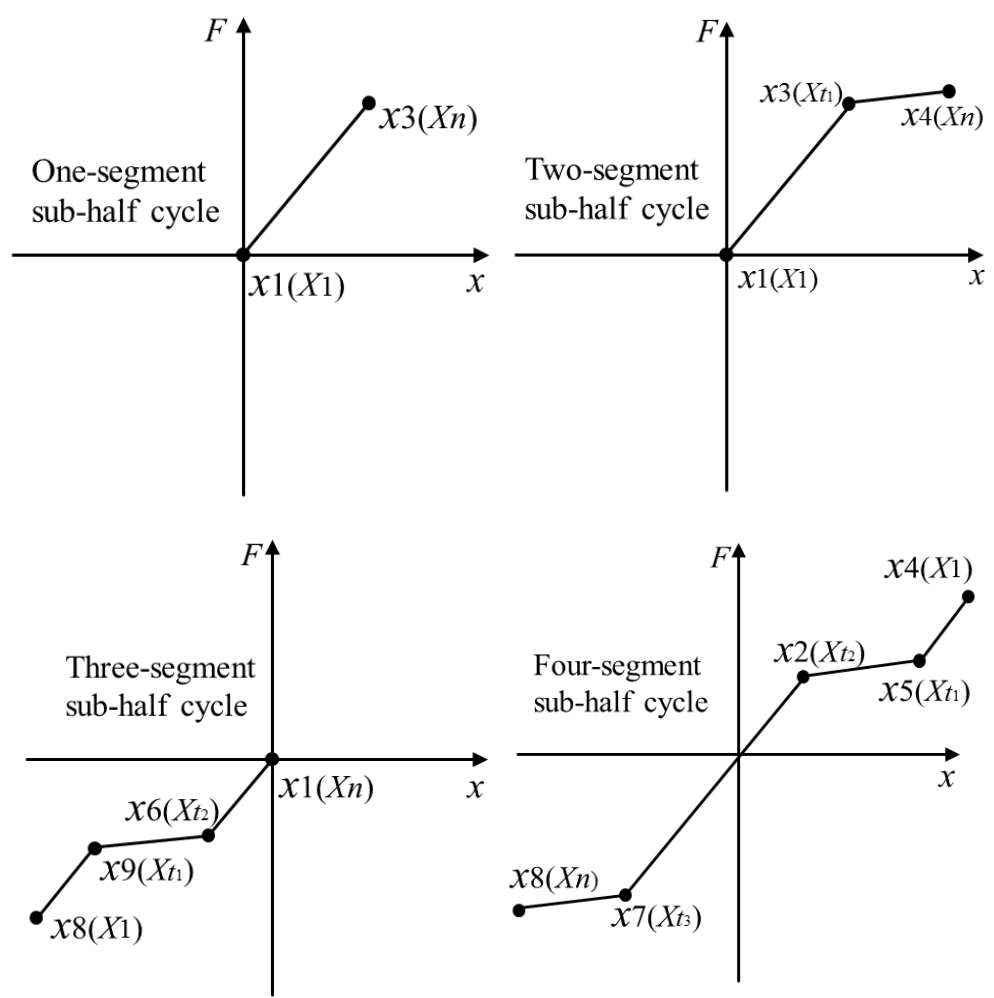

(b)

Fig. 1 (a) Idealized flag-shaped hysteretic loop, (b) with four types of possible half cycles for $r=1,2,3,4$. 


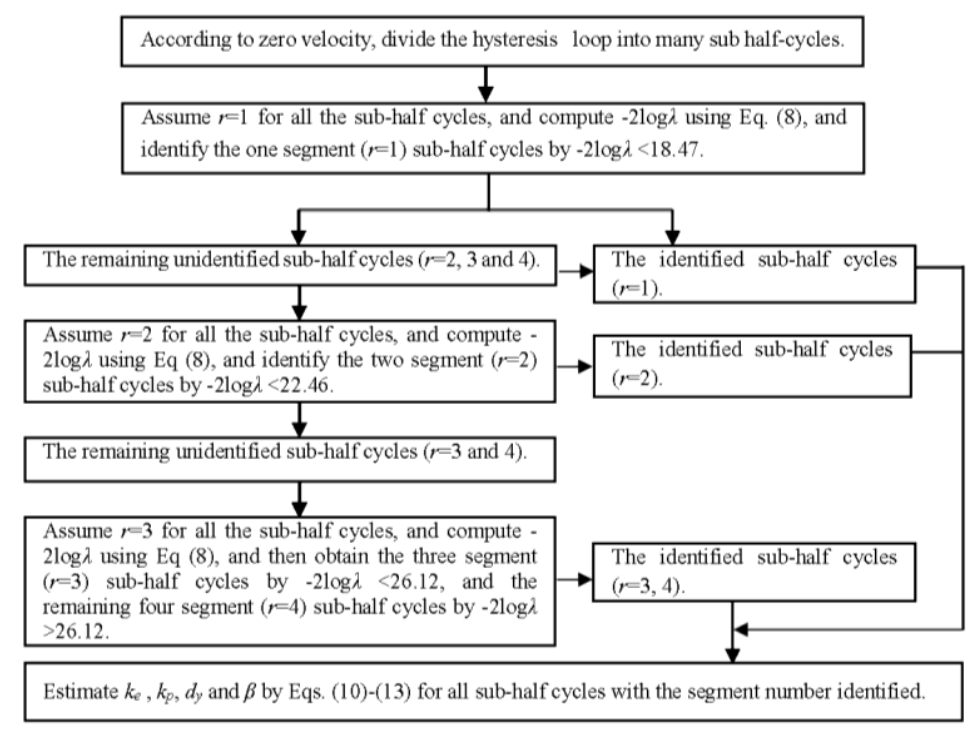

Fig. 2 Flowchart of the algorithm of the identification procedure. 


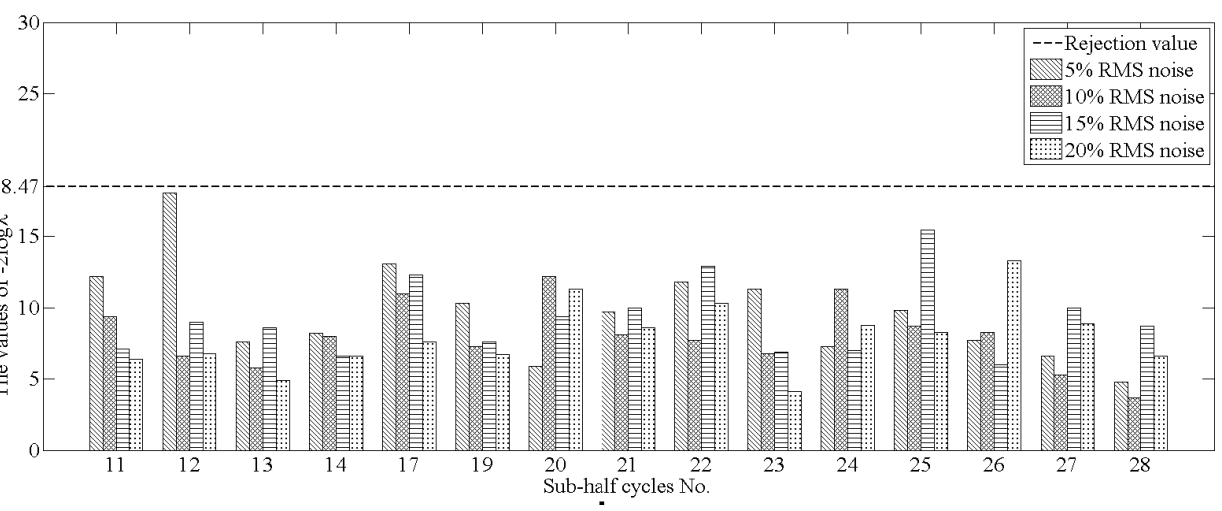

(a) one segment $(r=1)$ sub-half cycles

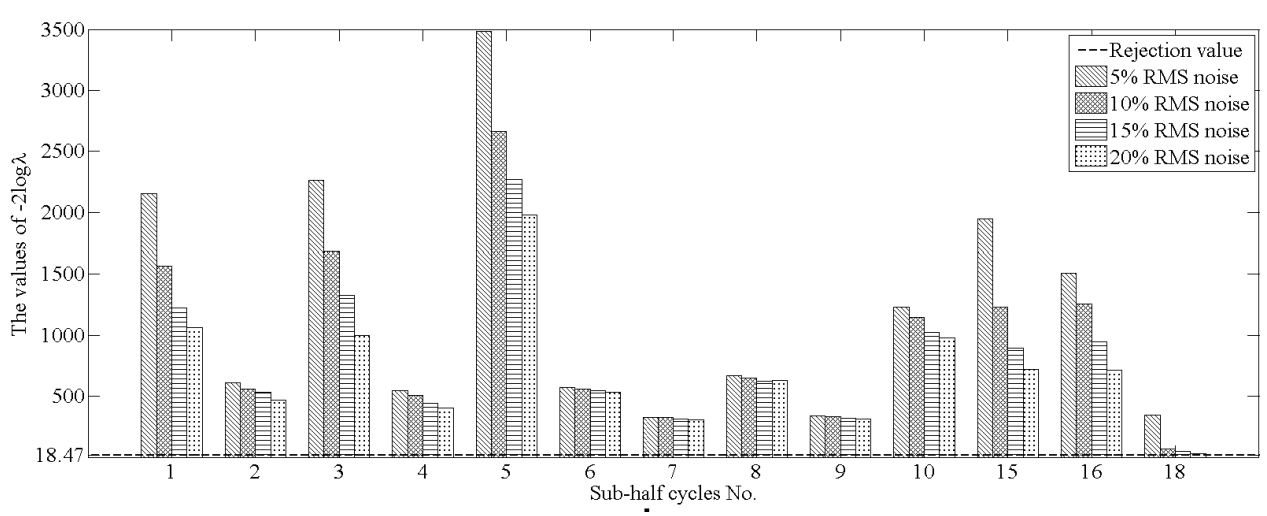

(b) more than one segment $(r=2,3$ and 4$)$ sub-half cycles

Fig. 3 Identification of one segment ( $r=1)$ sub-half cycles with variable noise level: (a) one segment sub-half cycles with $-2 \log \lambda<18.47$, (b) more than one segment sub-half cycles with $-2 \log \lambda>18.47$. 


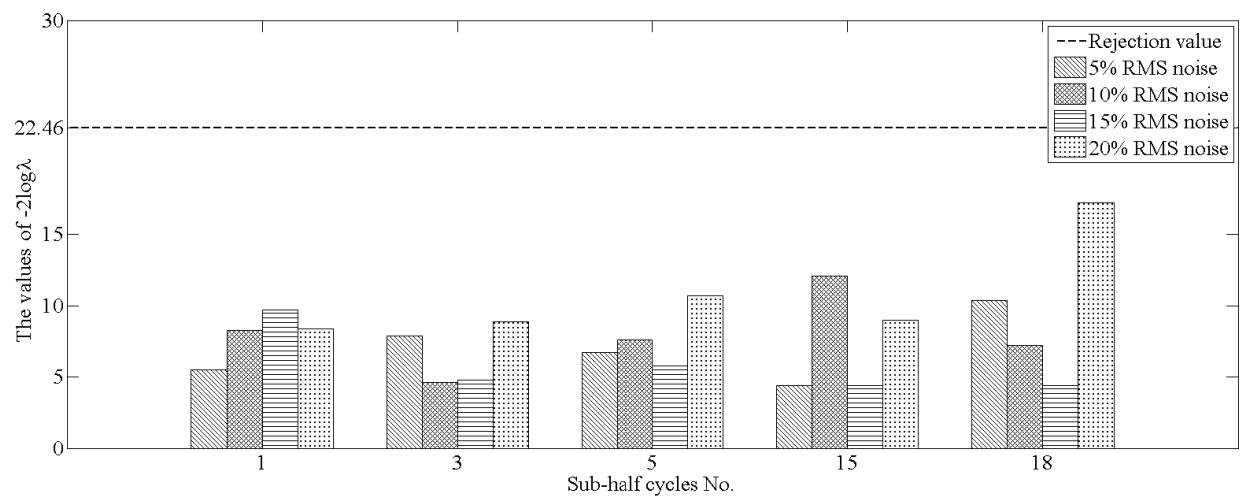

(a) two segment $(r=2)$ sub-half cycles

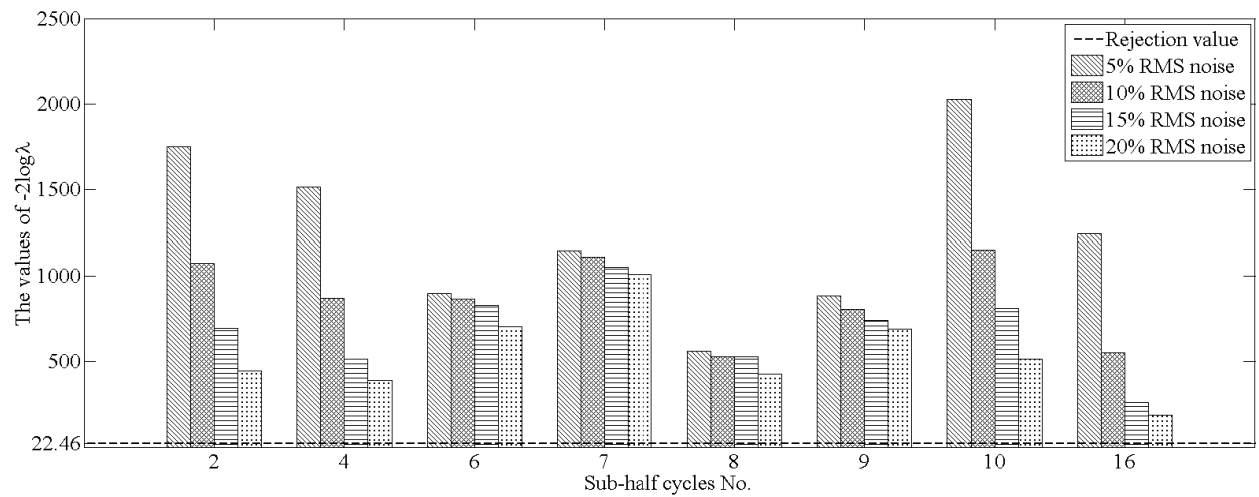

(b) more than two segment ( $r=3$ and 4) sub-half cycles

Fig. 4 Identification of two segment $(r=2)$ sub-half cycles, excluding the sub-half cycles found to be one segment: (a) two segment sub-half cycles with $-2 \log \lambda<22.46$, (b) more than two segment sub-half cycles with $-2 \log \lambda>22.46$. 


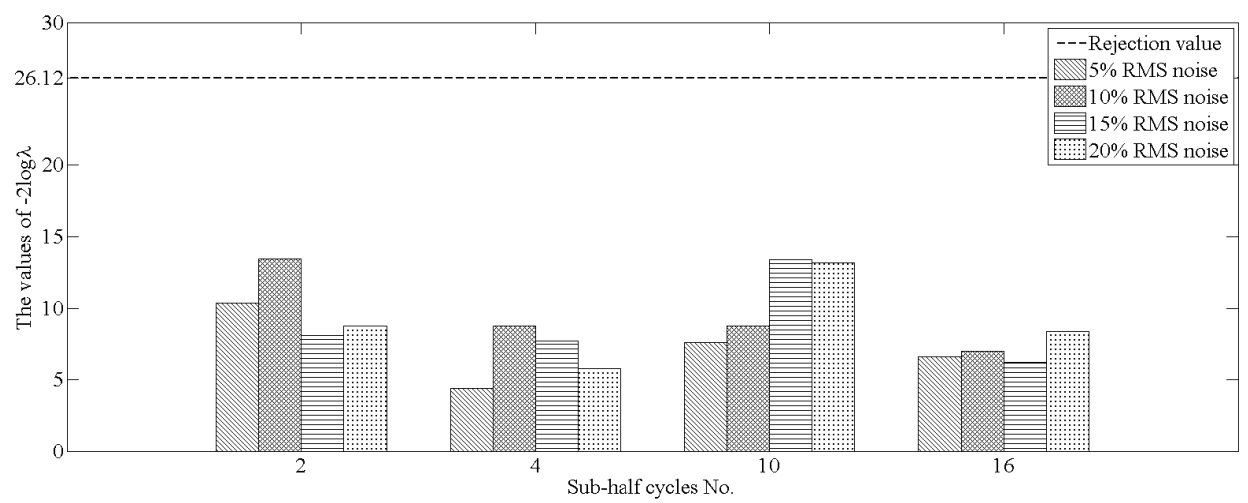

(a) three segment $(r=3)$ sub-half cycles

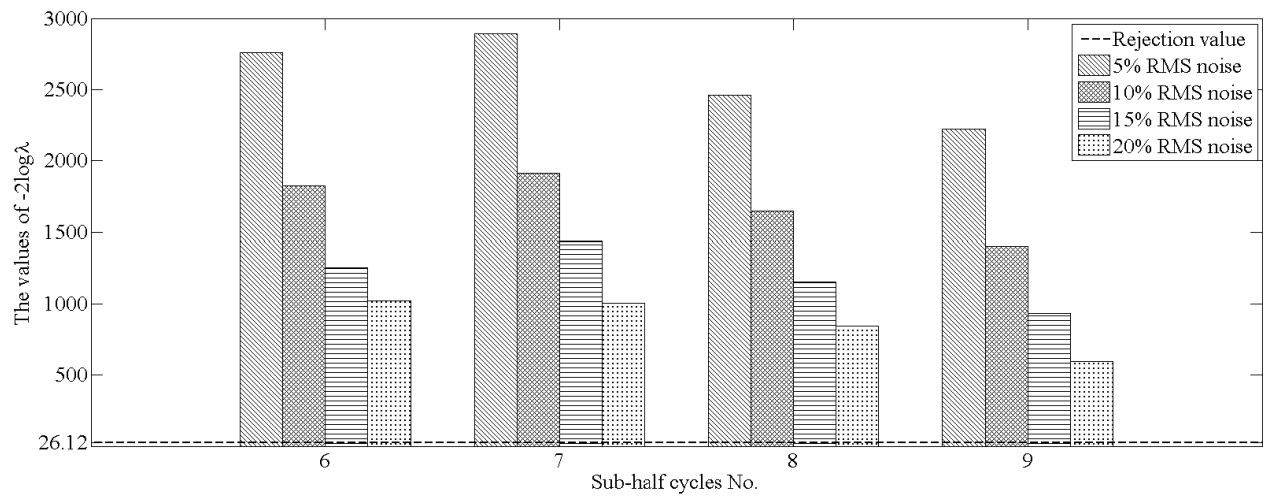

(b) four segment $(r=4)$ sub-half cycles

Fig. 5 Identification of three segment $(r=3)$ sub-half cycles, excluding one and two segment sub-half cycles: (a) three segment sub-half cycles with $-2 \log \lambda<26.12$, (b) four segment half cycles with $-2 \log \lambda>26.12$. 


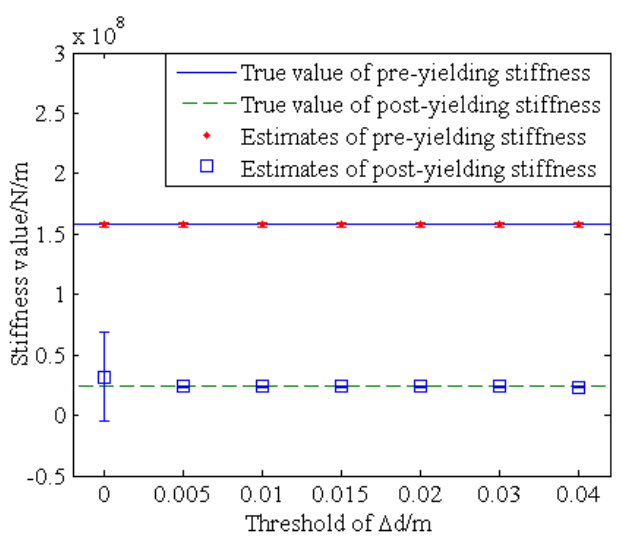

(a) $5 \%$ RMS noise

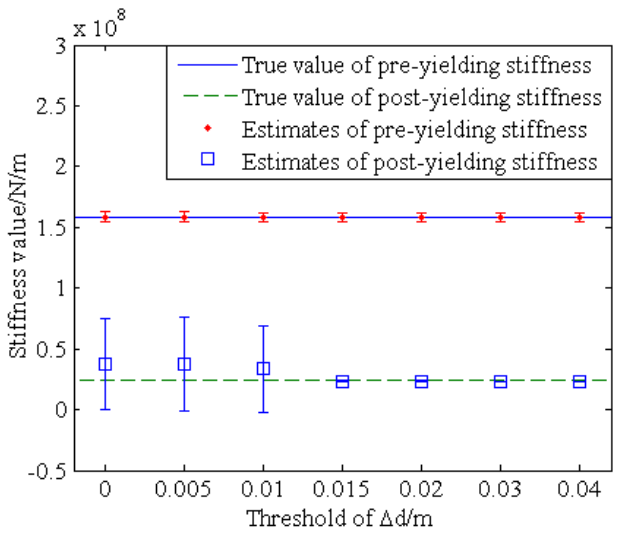

(c) $15 \%$ RMS noise

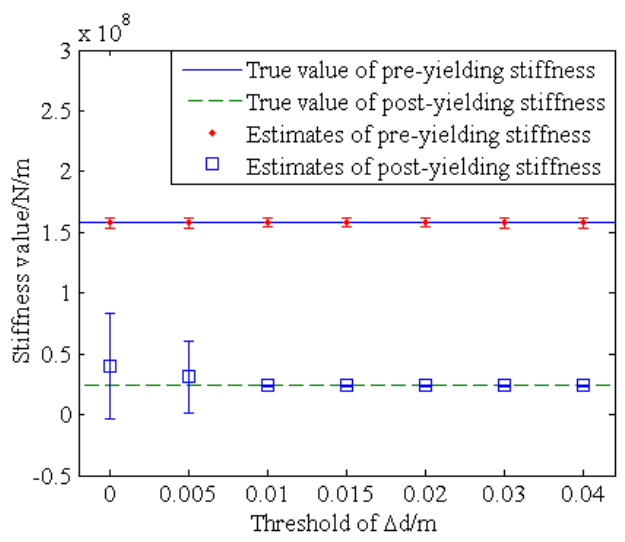

(b) $10 \%$ RMS noise

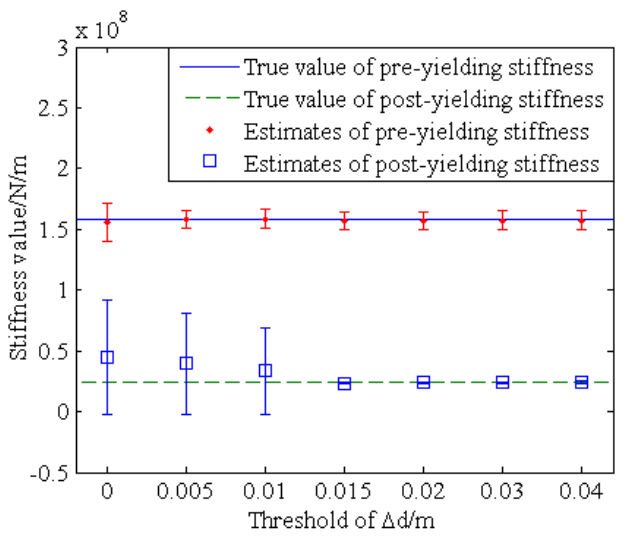

(d) $20 \%$ RMS noise

Fig. 6 Estimates results of stiffness, (a) at 5\% RMS noise, (b) at 10\% RMS noise, (c) at 15\% RMS noise, (d) at $20 \%$ RMS noise. The error bars show the mean and one standard deviation over all sub-half cycles. 


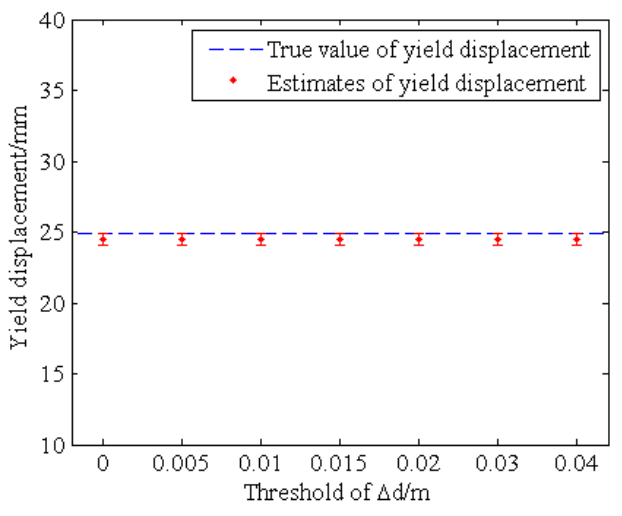

(a) $5 \%$ RMS noise

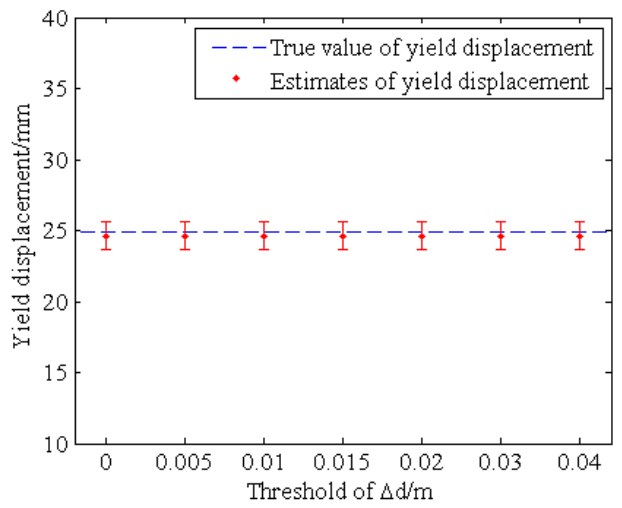

(c) $15 \%$ RMS noise

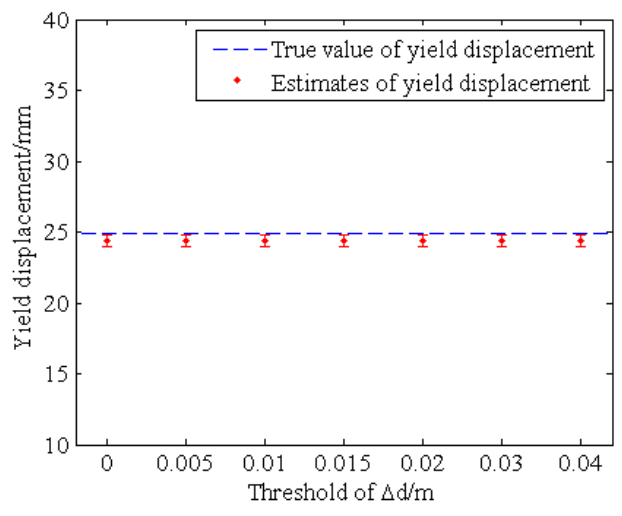

(b) $10 \%$ RMS noise

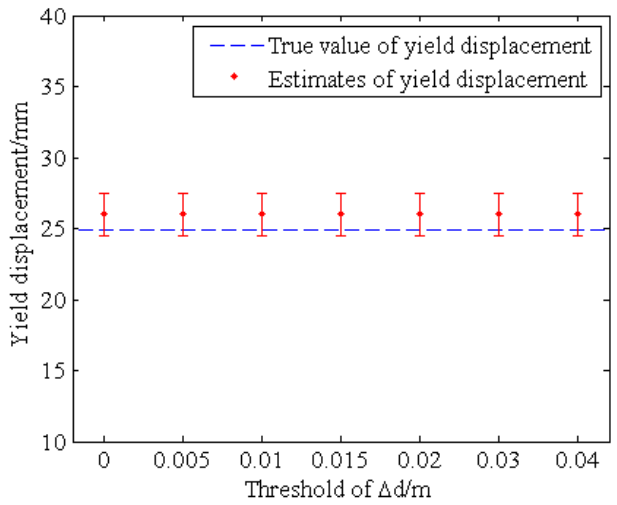

(d) $20 \%$ RMS noise

Fig. 7 Estimates results of yield displacement, (a) at 5\% RMS noise, (b) at 10\% RMS noise, (c) at 15\% RMS noise, (d) at $20 \%$ RMS noise. 


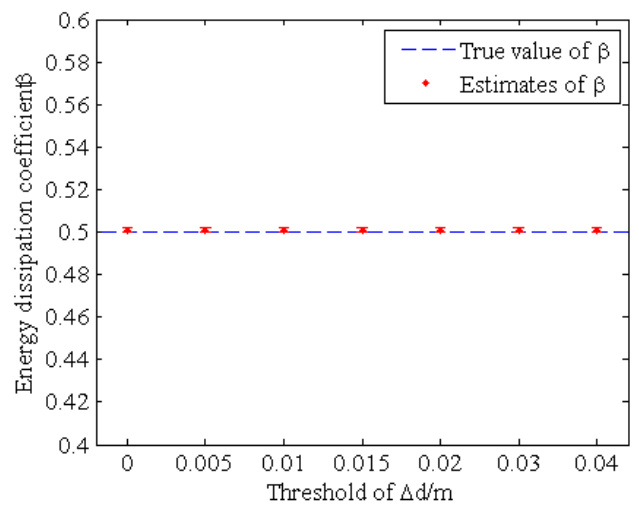

(a) 5\% RMS noise

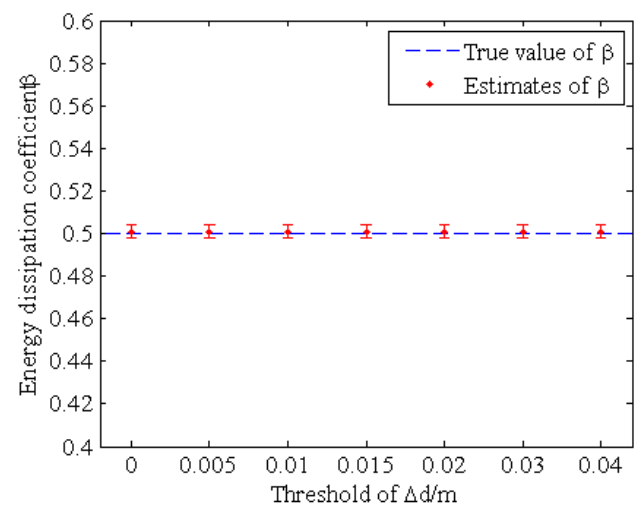

(c) 15\% RMS noise

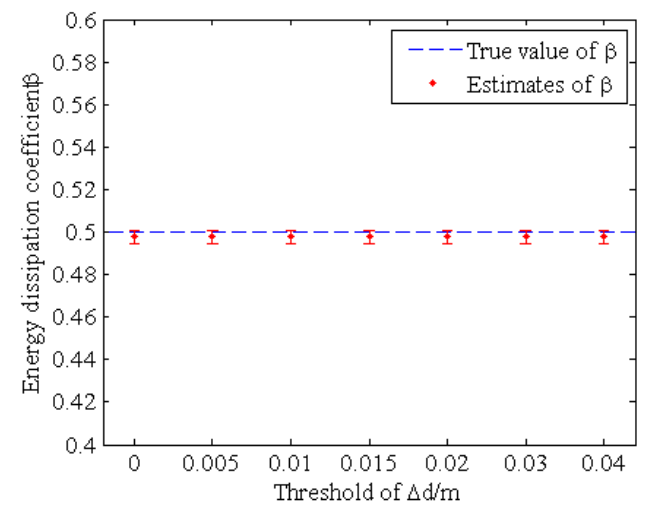

(b) $10 \%$ RMS noise

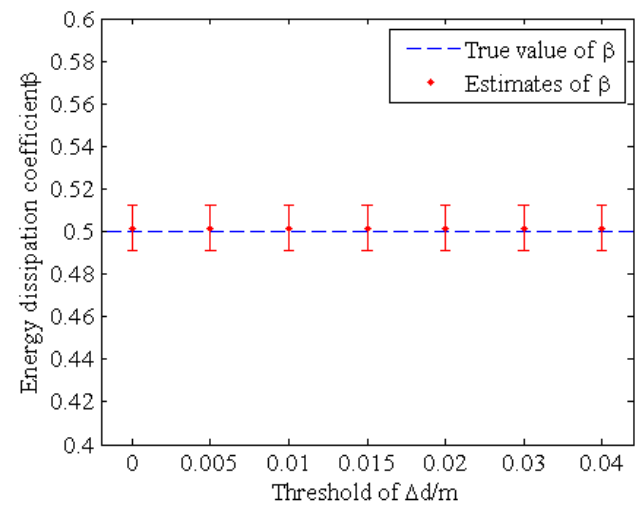

(d) $20 \%$ RMS noise

Fig. 8 Estimates results of $\beta$, (a) at 5\%, (b) 10\%, (c) 15\%, and (d) 20\% RMS noise. 


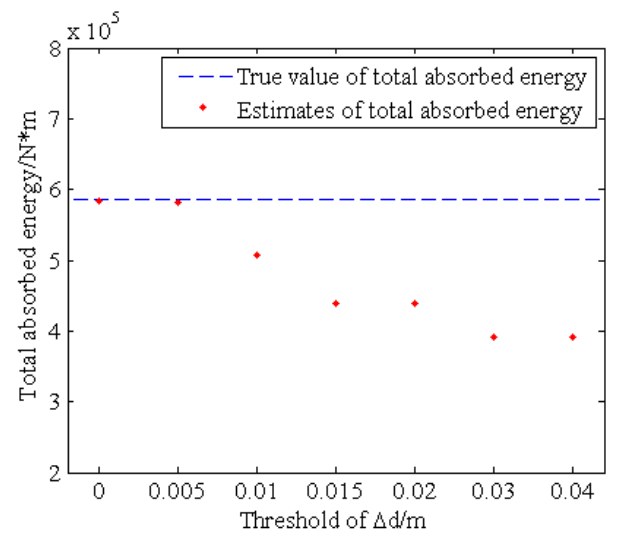

(a) $5 \%$ RMS noise

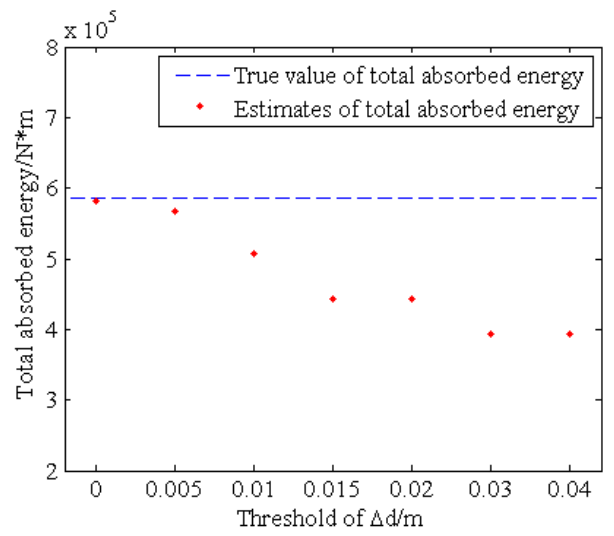

(c) $15 \%$ RMS noise

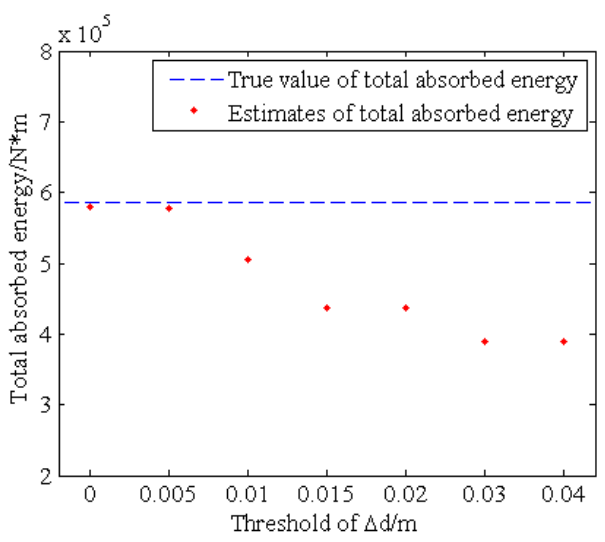

(b) $10 \%$ RMS noise

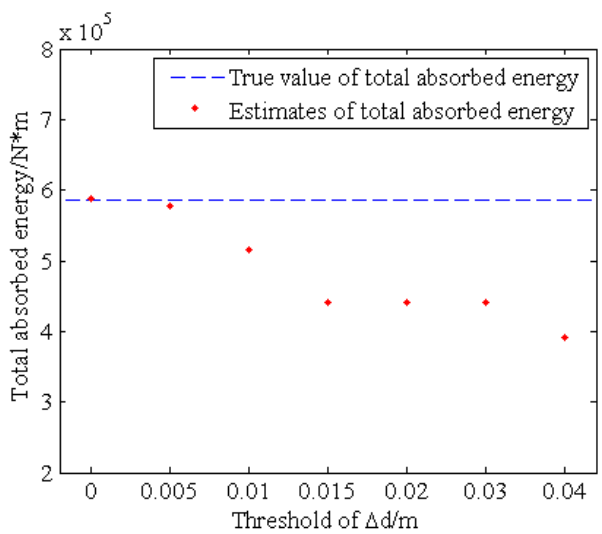

(d) $20 \%$ RMS noise

Fig. 9 Estimates results of total absorbed energy, (a) at 5\% RMS noise, (b) at 10\% RMS noise, (c) at 15\% RMS noise, (d) at $20 \%$ RMS noise. 